\title{
Multiphysics Platform for Simulation of Concrete Degradation
}

Benjamin W. Spencer, Hai Huang, Amit Jain, Mohamed Abdelrahman, Bora Gencturk, Yunping Xi

March 2018

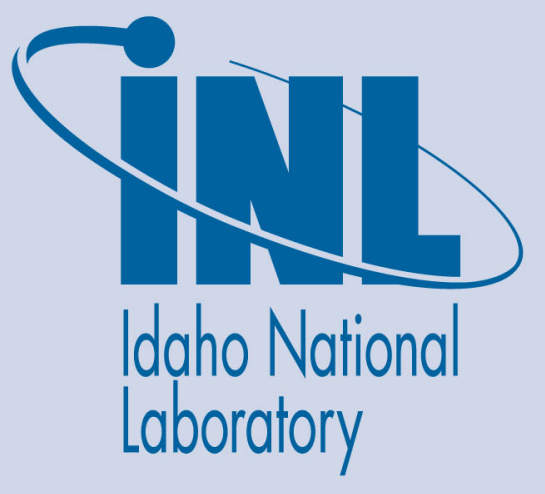

The INL is a U.S. Department of Energy National Laboratory operated by Battelle Energy Alliance 


\title{
Multiphysics Platform for Simulation of Concrete Degradation
}

\author{
Benjamin W. Spencer, Hai Huang, Amit Jain, Mohamed Abdelrahman, Bora \\ Gencturk, Yunping Xi
}

March 2018

Idaho National Laboratory Idaho Falls, Idaho 83415

http://www.inl.gov

Prepared for the

U.S. Department of Energy

Under DOE Idaho Operations Office

Contract DE-AC07-05ID14517 


\title{
Multiphysics Platform for Simulation of Concrete Degradation
}

\author{
Milestone Report \\ NEUP Project DE-FOA-0001129 \\ March, 2018
}

\author{
Benjamin W. Spencer ${ }^{1}$, Hai Huang ${ }^{1}$, Amit Jain ${ }^{2}$, Mohamed Abdelrahman ${ }^{3}$ \\ Bora Gencturk ${ }^{2}$, Yunping $\mathrm{Xi}^{3}$ \\ ${ }^{1}$ Idaho National Laboratory \\ Idaho Falls, ID 83415-3840 \\ ${ }^{2}$ University of Southern California \\ Los Angeles, CA 90089 \\ ${ }^{3}$ University of Colorado \\ Boulder, CO 80309
}




\section{Introduction}

The present NEUP project is focused on developing computational tools and experimental validation data for prediction of concrete degradation due to multiple mechanisms. The end goal of this work is to deliver validated models of concrete degradation processes and their effects on structures, which can be used to support lifetime extension efforts for operating U.S. commercial nuclear power plants.

This report documents the development of computational tools for this purpose in the Grizzly multiphysics simulation environment. The Grizzly code has been under development for several years at Idaho National Laboratory (INL) to address a variety of aging mechanisms in nuclear power plants, including concrete. Grizzly is based on MOOSE, an open-source multiphysics framework for solving coupled systems of partial differential equations.

Recently, a new simulation code called BlackBear has been released as open-source software by INL. BlackBear contains the portions of Grizzly that are generally applicable to all concrete structures, and not specifically to nuclear power plants. Grizzly now inherits its concrete modeling capabilities from BlackBear, so any capabilities added to BlackBear are automatically also available in Grizzly. This software was made open source to facilitate collaboration and taking contributions from the broader worldwide concrete modeling community. All of the models documented in the report have been developed in the BlackBear, and have either been merged into the main BlackBear source code repository, or some cases as noted, are in the process of review for merging into this code base.

This project has developed capabilities for nonlinear mechanical constitutive modeling of concrete, and for simulation of multiple interacting chemical species involved in important concrete degradation mechanisms, coupled with heat and moisture transport models. Section 2 documents the development of two nonlinear mechanical constitutive models implemented in BlackBear: a simple damage mechanics model, and a more comprehensive damaged plasticity model. Section 3 documents the capabilities for coupled species transport implemented in BlackBear based on the Nernst-Planck equations. Much of this work was already completed in previous years of this project and has already been documented in detail in the Fiscal Year 2017 Annual Report for this project. The focus of this report is on the implementation of these models in BlackBear. Validation of these models is underway and will be covered in the Annual Report for this project. 


\section{Mechanical Constitutive Models}

Most degradation mechanisms of interest in concrete involve expansion in regions of the structure, which can be due to a number of phenomena. Because this expansion is typically nonuniform spatially, and the concrete is typically confined with reinforcing bars, this expansion causes stresses, which can be high enough to induce damage. Damage can affect the transport of the species, moisture, and heat that were the source of the expansion, so it is important to have a robust capability for modeling volumetric expansion and damage as part of a comprehensive simulation capability for concrete degradation.

Outside this project, significant effort has been put into developing models in BlackBear for expansive strains due to alkali-silica reaction, and for creep. This project is developing models for damage that can be used in conjunction with these existing models. In MOOSE, the mechanical constitutive modeling capability was originally provided by a module called SolidMechanics. For a number of reasons, this module is being replaced by a new module called TensorMechanics, which is more general and modular.

Because the models need to be fully interoperable, they must be developed on a common platform. The alkali-silica reaction expansion model was originally developed based on SolidMechanics, but has been migrated to a new implementation based on TensorMechanics as part of the present work.

This project has developed two nonlinear constitutive models for concrete. To be interoperable with all other models, these models are also based on TensorMechanics. The two models are a simple damage model based on a formulation by Mazars, and a more comprehensive model based on the Lee and Fenves model, that includes effects represented by both damage and plasticity. These models are described below. In addition, this project has developed a shrinkage model, also based on TensorMechanics.

\subsection{Mazars Damage Model}

The Mazars model is based on a theory of elasticity coupled with the isotropic damage. It describes the elastic stiffness degradation and the softening behavior of concrete under both uniaxial tension and uniaxial compression using an isotropic scalar damage variable $w$. The damage depends only on the effective stresses applied to an undamaged area. The evolution of the damage is determined by the equivalent strain that quantifies the amount of the local extension state during the mechanical loading. It is calculated from the positive eigenvalues of the strain tensor as follows:

$$
\varepsilon=\sqrt{\sum_{i=1}^{3}\left(\left\langle\varepsilon_{i}\right\rangle_{+}\right)^{2}}
$$

where $\varepsilon_{i}$ is the principal strain, \langle\rangle$_{+}$is the Macaulay bracket and is defined so that: 


$$
\begin{cases}\left\langle\varepsilon_{i}\right\rangle_{+}=\varepsilon_{i} & \varepsilon_{i} \geq 0 \\ \left\langle\varepsilon_{i}\right\rangle_{+}=0 & \varepsilon_{i} \leq 0\end{cases}
$$

The loading damage threshold is defined as a function of equivalent strain as follows:

$$
f(\varepsilon, \kappa)=\varepsilon-\kappa
$$

where $k$ is the parameter-containing threshold of damage growth. Initially, $k$ is equal to $f_{t} / E_{o}$ and then it takes the maximum value of the equivalent strain during the entire load history

$$
\kappa_{d}=\max \left(\varepsilon, \kappa_{o}\right)
$$

In order to account for the different behavior of the concrete in tension and in compression, the scalar damage $w$ is defined as a combination of two damaging modes as expressed in:

$$
w=\alpha_{t} w_{t}+\alpha_{c} w_{c}
$$

where $\alpha$ t and $\alpha c$ are dimensionless coefficients and represent the contribution of each loading mode such that $\alpha \mathrm{t}=1$ and $\alpha \mathrm{c}=0$ under uniaxial tension; and, $\alpha \mathrm{t}=0$ and $\alpha \mathrm{c}=1$ under uniaxial compression. These coefficients are defined as functions of the strain that results from negative and positive stresses:

$$
\begin{aligned}
& \varepsilon_{k l}{ }^{t}=(1-w) C_{i j k l}{ }^{-1} \sigma_{i j}{ }^{t} \\
& \varepsilon_{k l}{ }^{c}=(1-w) C_{i j k l}{ }^{-1} \sigma_{i j}{ }^{c} \\
& \alpha_{t}=\sum_{i=1}^{3}\left(\frac{\left\langle\varepsilon_{i}^{t}\right\rangle\left\langle\varepsilon_{i}\right\rangle}{\varepsilon^{2}}\right)^{\beta} \\
& \alpha_{c}=\sum_{i=1}^{3}\left(\frac{\left\langle\varepsilon_{i}^{c}\right\rangle\left\langle\varepsilon_{i}\right\rangle}{\varepsilon^{2}}\right)^{\beta}
\end{aligned}
$$

where $w t$ and $w c$ are the scalar damage variables for tension and compression, respectively.

$$
\begin{aligned}
& w_{t}=1-\frac{\kappa_{o}\left(1-A_{t}\right)}{\kappa}-\frac{A_{t}}{\exp \left[B_{t}\left(\kappa-\kappa_{o}\right)\right]} \\
& w_{c}=1-\frac{\kappa_{o}\left(1-A_{c}\right)}{\kappa}-\frac{A_{c}}{\exp \left[B_{c}\left(\kappa-\kappa_{o}\right)\right]}
\end{aligned}
$$

where $A_{t}, A_{c}, B_{t}$, and $B_{c}$, are material parameters that control the shape of the curve peak and are determined using compression and traction tests. 


\subsection{Plastic Damage Model for Concrete}

\subsubsection{Introduction}

Researchers (Lubliner et al. 1989, Lee and Fenves 1998, Imran and Pantazopoulou 2001, Cicekli et al. 2007) have proposed different material models for concrete to characterize the effect of tensile and compressive damage along with the degradation of the elastic stiffness. Lee and Fenves (1998) proposed a model that decouples the effect of continuum damage of elastic stiffness and yield strength based on the concepts of fracture energy. Thus, plastic damage model for concrete by Lee and Fenves (1998) is chosen here for the implementation of material model for concrete in MOOSE. The material model will be used to study the crack initiation and crack propagation in concrete due to various mechanical loading conditions such as corrosion of steel reinforcement.

\subsubsection{Plastic damage material model for concrete}

The plastic damage model for concrete by Lee (1997), Lee and Fenves (1998), (2001) considers the damage of continuum elastic stiffness of material separately in tension and compression. Effect of crack opening and closing on the elastic stiffness during cyclic loading is included in the model.

Implementation of the material model is here done in two main steps. In the first step, the calculations are done in the effective stress space and in the second step, the effective stress is effective stress space, yield function, plastic potential, yield stresses, and hardening potential are provided in the following sub-sections.

\subsubsection{Effective stress space}

Using incremental theory of plasticity, the total strain tensor can be decomposed into elastic and plastic strain as follows:

$$
\epsilon=\epsilon^{e}+\epsilon^{p}
$$

where $\epsilon$ is total strain tensor, $\epsilon^{\boldsymbol{e}}$ is elastic strain tensor and $\epsilon^{p}$ is plastic strain tensor. The relation between elastic strain tensor and stress tensor, $\boldsymbol{\sigma}$, is given as

$$
\epsilon^{e}=\mathbb{E}^{-1}: \sigma
$$

where $\mathbb{E}$ is elasticity matrix. Using Eq. (12) and Eq.(13), the relation between the plastic strain tensor and stress tensor, $\boldsymbol{\sigma}$, is expressed as

$$
\sigma=\mathbb{E}:\left(\epsilon-\epsilon^{p}\right)
$$

As mentioned earlier, the model considered the effect of damage of elastic stiffness, hence, an effective stress space, $\overline{\boldsymbol{\sigma}}$, is defined, where stress for a given strain always corresponds to the undamaged elastic stiffness. The relation between effective stress tensor and strain tensor is

$$
\overline{\boldsymbol{\sigma}}=\mathbb{E}_{\mathbf{0}}:\left(\boldsymbol{\epsilon}-\boldsymbol{\epsilon}^{p}\right)
$$

where $\mathbb{E}_{\mathbf{0}}$ is the undamaged elasticity matrix of the material. To consider the degradation of reinforced-concrete structures, an isotropic damage in considered in concrete material. Hence, 
the relation between the effective stress space and the stress space can be established by an isotropic scalar degradation damage variable, $D$, as

$$
\begin{gathered}
\boldsymbol{\sigma}=(1-D) \overline{\boldsymbol{\sigma}} \\
\boldsymbol{\sigma}=(1-D) \mathbb{E}_{\mathbf{0}}:\left(\boldsymbol{\epsilon}-\boldsymbol{\epsilon}^{p}\right)
\end{gathered}
$$

\subsubsection{Yield function}

Strength of material in tension and compression is expressed in terms of damage variable as

$$
\begin{aligned}
& f_{t}=f_{t}\left(\kappa_{t}\right) \\
& f_{c}=f_{c}\left(\kappa_{c}\right)
\end{aligned}
$$

where $\kappa_{t}$ and $\kappa_{c}$ are the damage variables in tension and compression, respectively. The yield function is used to describe the admissible stress space, in terms of the stress space and the strength corresponding to that space. For this implementation, the yield function is defined in the stress space as follows:

$$
\mathcal{F}\left(\boldsymbol{\sigma}, f_{t}, f_{c}\right)=\frac{1}{1-\alpha}\left(\alpha I_{1}+\sqrt{J_{2}}+\beta<\hat{\sigma}_{\max }>\right)-f_{c}(\boldsymbol{\kappa})
$$

where,

$I_{1} \quad: \quad$ first invariant of the stress tensor

$J_{2} \quad$ : $\quad$ second invariant of deviatoric component of the stress tensor

$\hat{\sigma}_{\max }: \quad$ algebraically maximum principal stress

$\alpha \quad: \quad$ material parameter $\left(=\frac{f_{b 0}-f_{c 0}}{2 f_{b 0}-f_{c 0}}\right)$

$f_{t 0} \quad$ : $\quad$ yield strength of concrete in uniaxial tensile loading

$f_{b 0} \quad$ : $\quad$ yield strength of concrete in biaxial compressive loading

$f_{c 0} \quad: \quad$ yield strength of concrete in uniaxial compressive loading

$\beta \quad: \quad$ material parameter $\left(=\frac{f_{c 0}}{f_{t 0}}(\alpha-1)-(1+\alpha)\right)$

$f_{t}, f_{c}: \quad$ yield strength of concrete in tension and compression, respectively

$\boldsymbol{\kappa}:$ a vector of damage variables $\left(=\left\{\kappa_{t}, \kappa_{c}\right\}\right)$

As mentioned earlier that the implementation first obtains the effective stress, hence, the yield stress of the material in uniaxial loading is expressed in terms of the effective yield stress as

$$
\begin{aligned}
& f_{t}=\left(1-D_{t}\left(\kappa_{t}\right)\right) \bar{f}_{t}\left(\kappa_{t}\right) \\
& f_{c}=\left(1-D_{c}\left(\kappa_{c}\right)\right) \bar{f}_{c}\left(\kappa_{c}\right)
\end{aligned}
$$

where $\bar{f}_{t}$ and $\bar{f}_{c}$ are the yield strength of material in tension and compression in the effective stress space, respectively, and $0 \leq D_{t}<1$ and $0 \leq D_{c}<1$ are degradation damage responses in tension and compression, respectively. Earlier mentioned scalar degradation damage variable, $D$, is expressed as 


$$
D=D(\kappa)=1-\left(1-D_{t}\left(\kappa_{t}\right)\right)\left(1-D_{c}\left(\kappa_{c}\right)\right)
$$

Hence, for uniaxial tension, $D=D_{t}$, while for uniaxial compression, $D=D_{c}$. Thus, yield stress in uniaxial loading, i.e. Eq.(21) and Eq. (22), can be rewritten as

$$
\begin{aligned}
& f_{t}=(1-D) \bar{f}_{t}\left(\kappa_{t}\right) \\
& f_{c}=(1-D) \bar{f}_{c}\left(\kappa_{c}\right)
\end{aligned}
$$

The yield function, $\mathcal{F}\left(\boldsymbol{\sigma}, f_{t}, f_{c}\right)$, is a homogenous function, i.e. $x \mathcal{F}\left(\boldsymbol{\sigma}, f_{t}, f_{c}\right)=\mathcal{F}\left(x \boldsymbol{\sigma}, x f_{t}, x f_{c}\right)$, hence, yield function in the effective stress space is obtained by multiplying by a factor $(1-D)$ of both sides of Eq.(18) to obtain

$$
\mathcal{F}\left(\widehat{\boldsymbol{\sigma}}, \overline{f_{t}}, \overline{f_{c}}\right)=\frac{1}{1-\alpha}\left(\alpha \overline{I_{1}}+\sqrt{3 \overline{J_{2}}}+\beta(\boldsymbol{\kappa})<\hat{\bar{\sigma}}_{\text {max }}>\right)-\bar{f}_{c}\left(\kappa_{c}\right)
$$

where,

$$
\begin{array}{lll}
\widehat{\overline{\boldsymbol{\sigma}}} & : & \text { eigenvalues of effective stress tensor } \\
\bar{I}_{1} & : & \text { first invariant of the effective stress tensor } \\
\bar{J}_{2} & : & \text { second invariant of deviatoric component of the effective stress tensor } \\
\overline{\bar{\sigma}}_{\text {max }}: & \text { algebraically maximum principal effective stress } \\
\beta(\kappa) & : & \text { material parameter }\left(=\frac{\bar{f}_{c}\left(\kappa_{c}\right)}{\bar{f}_{t}\left(\kappa_{t}\right)}(\alpha-1)-(1+\alpha)\right)
\end{array}
$$

\subsubsection{Plastic potential}

In the effective stress space, the plastic potential governs the plastic strain rate, $\dot{\epsilon}^{p}$ which is given by

$$
\begin{gathered}
\dot{\epsilon}^{p}=\dot{\lambda} \nabla_{\sigma} \boldsymbol{\Phi}(\widehat{\overline{\boldsymbol{\sigma}}}) \\
\boldsymbol{\Phi}(\widehat{\overline{\boldsymbol{\sigma}}})=\alpha_{p} \overline{I_{1}}+\|\overline{\boldsymbol{s}}\|
\end{gathered}
$$

where $\boldsymbol{\Phi}$ is the plastic potential function, $\|\overline{\mathbf{s}}\|$ is the norm of the effective deviatoric stress tensor, $\alpha_{p}$ is a parameter that govern dilatancy, and $\dot{\lambda}$ is the plastic consistency parameter. $\dot{\lambda}$ and $\mathcal{F}\left(\overline{\boldsymbol{\sigma}}, \bar{f}_{t}, \bar{f}_{c}\right)$ satisfy the following Kuhn-Tucker conditions

$$
\begin{gathered}
\dot{\lambda} \geq 0, \\
\dot{\lambda} \dot{\mathcal{F}}\left(\widehat{\overline{\boldsymbol{\sigma}}}, \bar{f}_{t}, \bar{f}_{c}\right)=0
\end{gathered}
$$

\subsubsection{Yield stress of material}

To express the strain hardening behavior in compression and strain softening in tension, the effective yield stress of material and degradation damage variables are expressed as follows 


$$
\begin{aligned}
& \bar{\sigma}_{N}=f_{N 0}\left(\frac{\left(1+a_{N}\right)-\sqrt{\Phi_{N}\left(\kappa_{N}\right)}}{a_{N}}\right)^{1-\left(d_{N} / b_{N}\right)} \\
& D_{N}=1-\left(\frac{\left(1+a_{N}\right)-\sqrt{\Phi_{N}\left(\kappa_{N}\right)}}{a_{N}}\right)^{\left(d_{N} / b_{N}\right)}
\end{aligned}
$$

where $N=t$, for the uniaxial tension, $N=c$, for uniaxial compression, $a_{N}, b_{N}$, and $d_{N}$ are material constants, $\Phi_{N}\left(\kappa_{N}\right)=1+a_{N}\left(2+a_{N}\right) \kappa_{N}, f_{N 0}$ is initial yield stress of material and $\kappa_{N}$, is previously defined damage variable.

\subsubsection{Hardening potential}

A vector of two damage variables, $\boldsymbol{\kappa}=\left\{\begin{array}{ll}\kappa_{t} & \kappa_{c}\end{array}\right\}$, is used in the implementation as the state variable to consider the effect of damage in tension and compression, separately. The evolution of these damage variables is defined in terms of the hardening potential, $\mathbf{H}$, as

$$
\dot{\boldsymbol{\kappa}}=\dot{\lambda} \mathbf{H}(\widehat{\overline{\boldsymbol{\sigma}}}, \boldsymbol{\kappa})
$$

where, $\widehat{\hat{\boldsymbol{\sigma}}}$ is a vector of principal stresses. The evolution of the damage variable is expressed in terms of the evolution of plastic strain as follows:

$$
\dot{\kappa}_{N}=\frac{1}{g_{N}} \overline{f_{N}}\left(\kappa_{N}\right) \dot{\epsilon}^{p}
$$

where $g_{N}$ is dissipated energy density during the process of cracking and $N=t$ in tension and $N=c$ in compression. The scalar plastic strain rate, $\dot{\epsilon}^{p}$, is extended to multi-dimensional case as

$$
\dot{\epsilon}^{p}=\delta_{t N} r(\hat{\bar{\sigma}}) \dot{\epsilon}_{\max }^{p}+\delta_{c N}(1-r(\hat{\bar{\sigma}})) \dot{\epsilon}_{\min }^{p}
$$

where $\delta_{i j}=\left\{\begin{array}{ll}1 & i=j \\ 0 & i \neq j\end{array}\right\}$ is a delta function, $r(\hat{\bar{\sigma}})=\left\{\begin{array}{cc}0 & \text { if } \hat{\bar{\sigma}}=\mathbf{0} \\ \frac{\sum_{i=1}^{3}<\hat{\bar{\sigma}}_{i}>}{\sum_{i=1}^{3}\left|\widehat{\bar{\sigma}}_{i}\right|} & \text { otherwise }\end{array}\right\},<x>=\frac{(x+|x|)}{2}$ is the Macaulay bracket function, and $\dot{\epsilon}_{\max }^{p} / \dot{\epsilon}_{\min }^{p}$ are maximum and minimum principal plastic strains, respectively. From Eqs. (32) and (33), the evolution of the damage variables is obtained as

$$
\begin{gathered}
\dot{\boldsymbol{\kappa}}=\mathbf{h}(\widehat{\overline{\boldsymbol{\sigma}}}, \boldsymbol{\kappa}): \widehat{\hat{\boldsymbol{\epsilon}}^{\boldsymbol{p}}} \\
\boldsymbol{h}(\widehat{\overline{\boldsymbol{\sigma}}}, \boldsymbol{\kappa})=\left[\begin{array}{ccc}
\frac{r(\hat{\bar{\sigma}}) \bar{f}_{t}\left(\kappa_{t}\right)}{g_{t}} & 0 & 0 \\
0 & 0 & \frac{(1-r(\hat{\bar{\sigma}})) \bar{f}_{c}\left(\kappa_{c}\right)}{g_{c}}
\end{array}\right]
\end{gathered}
$$

':' represents products of two matrices. Hence, $\mathbf{H}(\widehat{\overline{\boldsymbol{\sigma}}}, \boldsymbol{\kappa})$ in Eq. (32) is obtained as follows:

$$
\mathbf{H}(\widehat{\overline{\boldsymbol{\sigma}}}, \boldsymbol{\kappa})=\mathbf{h} \cdot \nabla_{\widehat{\bar{\sigma}}} \boldsymbol{\Phi}(\widehat{\overline{\boldsymbol{\sigma}}})
$$


where '.' represents dot product of a matrix and a vector and $\nabla_{\widehat{\bar{\sigma}}}$ is gradient w.r.t. principal components of effective stress.

\subsubsection{Implementation in MOOSE}

\subsubsection{Flowchart}

In the model, the yield of material depends on pressure, deviatoric stress, and maximum principal stress in the material. Since, principal components of effective stress and plastic strain are frequently used in the above definitions. Three principal stress components are considered as three parameters to implement the model in MOOSE. The spectral decomposition of effective stress tensor, $\overline{\boldsymbol{\sigma}}$, is used to convert the second order tensor to a vector of principal components as follows:

$$
\widehat{\bar{\sigma}}=\xi^{T}: \overline{\boldsymbol{\sigma}}: \xi
$$

where, $\xi$ is a matrix of orthogonal eigenvectors. An existing return mapping algorithm in MOOSE is then used to obtain the effective stress, plastic strain and damage variables for a given displacement increment, see Section 2.8.2.2.

Figure 1 summarizes the procedure to obtain the unknown quantities in each step. As mentioned in the flow chart, the implementation first obtains solution in the effective stress space, $\widehat{\bar{\sigma}}$. The effective stresses are then mapped back to the stress space $\boldsymbol{\sigma}$, in the last step of implementation as per Eq.(16).

At first, the material is assumed to be elastic and $\overline{\boldsymbol{\sigma}}$ is obtained by assuming undamaged elastic stiffness, $\mathbb{E}_{\mathbf{0}}$. The yield function, $\mathcal{F}\left(\widehat{\overline{\boldsymbol{\sigma}}}, \bar{f}_{t}, \bar{f}_{c}\right)$, is checked for admissibility of the stress space. If $\widehat{\overline{\boldsymbol{\sigma}}}$ lies in the admissible domain, i.e., $\mathcal{F}\left(\widehat{\overline{\boldsymbol{\sigma}}}, \bar{f}_{t}, \bar{f}_{c}\right) \leq 0$, the material is in the elastic domain and the plastic strain and damage variables remain unchanged. If $\widehat{\bar{\sigma}}$ lies in the inadmissible domain, i.e., $\mathcal{F}\left(\widehat{\boldsymbol{\sigma}}, \bar{f}_{t}, \bar{f}_{c}\right)>0$, the material reaches its plastic domain. In this case, the $\widehat{\overline{\boldsymbol{\sigma}}}, \boldsymbol{\epsilon}$, and $\boldsymbol{\kappa}$ are obtained from the return mapping algorithm as described below. 


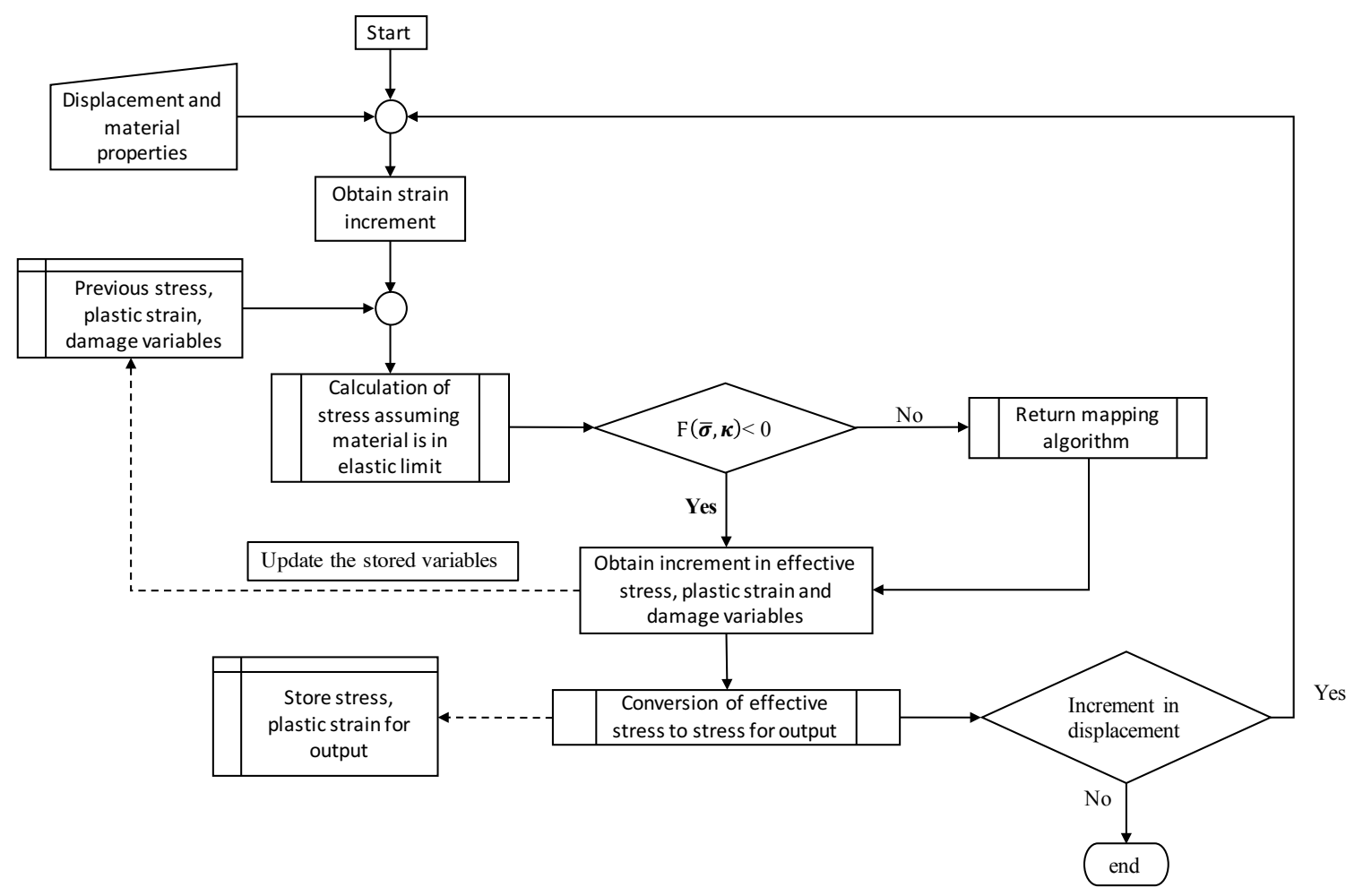

Figure 1: Flow chart to obtain the stresses for given displacement increment

\subsubsection{Return Mapping Algorithm}

In return mapping algorithm, the increment in the stress parameters, $\widehat{\widehat{\sigma}}$, and plastic multiplier, $\lambda$ are obtained in two steps. With the initial guess of $\widehat{\overline{\boldsymbol{\sigma}}}$ and $\lambda$, Newton-Raphson method is utilized with line search algorithm to obtain the converged solution of stress parameters and plastic multiplier. The L2 norm of residual vector, $\boldsymbol{r}$, defined as follows, is considered as a stopping criteria.

$$
\begin{gathered}
r_{i}=\widehat{\overline{\boldsymbol{\sigma}}}_{\boldsymbol{\imath}}-\widehat{\overline{\boldsymbol{\sigma}}}_{\boldsymbol{i}}^{\text {trial }}+\lambda \frac{d \Phi}{d \widehat{\boldsymbol{\sigma}}_{\boldsymbol{i}}} \forall \boldsymbol{i} \in\{1,2,3\} \\
r_{4}=\mathcal{F}(\widehat{\overline{\boldsymbol{\sigma}}}, \boldsymbol{\kappa})
\end{gathered}
$$

The converged solution of the stress parameters, $\widehat{\overline{\boldsymbol{\sigma}}}$, are rotated back to the effective stress, $\overline{\boldsymbol{\sigma}}$, configuration as follows:

$$
\overline{\boldsymbol{\sigma}}=\xi: \widehat{\bar{\sigma}}: \xi^{T}
$$

Effective stress and plastic multiplier are utilized to obtain the damage variables, $\boldsymbol{\kappa}$ as per Eq. (33). Thus, the stress space, $\boldsymbol{\sigma}$, is obtained using Eqs. (16) and (23).

\subsubsection{Results and discussion}

The implementation in MOOSE is validated for various loading cases at the material scale. At the material scale, a linear first order Lagrange eight-noded cubic element was considered as 
shown in Figure 2. Various loading conditions were applied whose boundary conditions are given in Table 1. Parameters used for the tests given mentioned in Table 2.

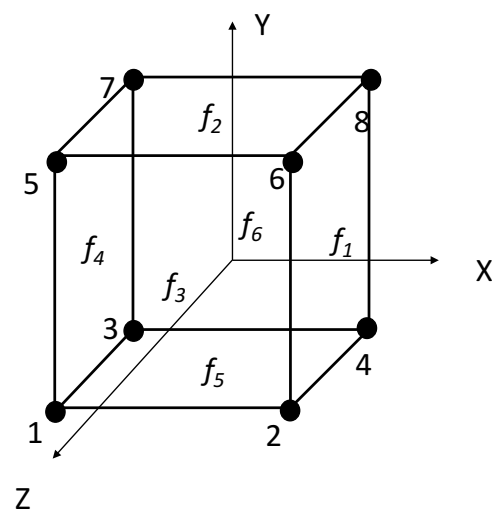

Figure 2: Eight-noded brick element

Table 1: Boundary conditions

\begin{tabular}{|c|c|}
\hline Loading case & Boundary conditions \\
\hline Uniaxial tension & $\begin{array}{r}u_{i x}=1 \times 10^{-6} x t \forall i \in\{1,2, \ldots, 8\}^{*} \\
u_{i y}=0 \forall i \in\{1,2,3,4\} \\
u_{i z}=0 \forall i \in\{3,4,7,8\}\end{array}$ \\
\hline Uniaxial compression & $\begin{array}{c}u_{i x}=-1 \times 10^{-5} x t \forall i \in\{1,2, \ldots, 8\} \\
u_{i y}=0 \forall i \in\{1,2,3,4\} \\
u_{i z}=0 \forall i \in\{3,4,7,8\}\end{array}$ \\
\hline Biaxial tension & $\begin{array}{c}u_{i x}=1 \times 10^{-6} x t \forall i \in\{1,2, \ldots, 8\} \\
u_{i y}=1 \times 10^{-6} y t \forall i \in\{1,2, \ldots, 4\} \\
u_{i z}=0 \forall i \in\{3,4,7,8\}\end{array}$ \\
\hline Biaxial compression & $\begin{array}{c}u_{i x}=-1 \times 10^{-5} x t \forall i \in\{1,2, \ldots, 8\} \\
u_{i y}=-1 \times 10^{-5} y t \forall i \in\{1,2, \ldots, 4\} \\
u_{i z}=0 \forall i \in\{3,4,7,8\}\end{array}$ \\
\hline Shear & $\begin{array}{c}p_{f_{i} x}=1 \times 10^{-3} t \forall i \in\{1,4\}^{* *} \\
p_{f_{i} y}=-1 \times 10^{-3} t \forall i \in\{2,5\} \\
u_{i z}=0 \forall i \in\{3,4,7,8\}\end{array}$ \\
\hline $\begin{array}{l}\text { Dilatancy in uniaxial } \\
\text { compression }\end{array}$ & $\begin{array}{c}u_{i x}=-1 \times 10^{-5} x t \forall i \in\{1,2, \ldots, 8\} \\
u_{i y}=0 \forall i \in\{1,2,3,4\} \\
u_{i z}=0 \forall i \in\{3,4,7,8\}\end{array}$ \\
\hline \multicolumn{2}{|c|}{$\begin{array}{l}{ }^{*} u_{i x}: \text { displacement on } i^{t h} \text { node in } x \text { direction } \\
{ }^{* *} p_{f_{i} x}: \text { pressure on face } f_{i} \text { in } x \text { direction }\end{array}$} \\
\hline
\end{tabular}

Table 2: Parameters used in test

\begin{tabular}{|l|c|l|}
\hline Parameters & Symbol & Value \\
\hline Elastic modulus of concrete & $E$ & $3.10 \mathrm{E}+04$ \\
\hline
\end{tabular}




\begin{tabular}{|l|c|l|} 
Poisson ratio for concrete & $v$ & 0.18 \\
\hline $\begin{array}{l}\text { Parameter relating biaxial compressive strength to uniaxial } \\
\text { compressive strength }\end{array}$ & $\alpha$ & 0.12 \\
\hline Parameter to control dilatancy behavior & $\alpha_{p}$ & 0.23 \\
\hline Yield strength in uniaxial tension (MPa) & $f_{t}$ & 3.48 \\
\hline Fracture energy in tension (N/m) & $G_{t}$ & 40 \\
\hline Yield strength in compression (MPa) & $f_{c}$ & -18.3 \\
\hline Fracture energy in compression (N/m) & $G_{c}$ & 5690 \\
\hline Damage at peak compressive stress in uniaxial compression & $D_{c}$ & 0.45 \\
\hline $\begin{array}{l}\text { Damage at half of the peak tensile stress after yielding in } \\
\text { uniaxial tension }\end{array}$ & $D_{t}$ & 0.65 \\
\hline Parameter used for yield strength in tension & $a_{t}$ & 0.001 \\
\hline Parameter used for yield strength in compression & $a_{c}$ & 3.8 \\
\hline
\end{tabular}

The results of the above-mentioned loading conditions are shown in Figure 3-8. It can be observed in all the figures that results from the implementation is very close to what obtained by Lee. However, the parameter used to control the dilatancy behavior has an offset of 0.055 , which may be due to lack information about the test.

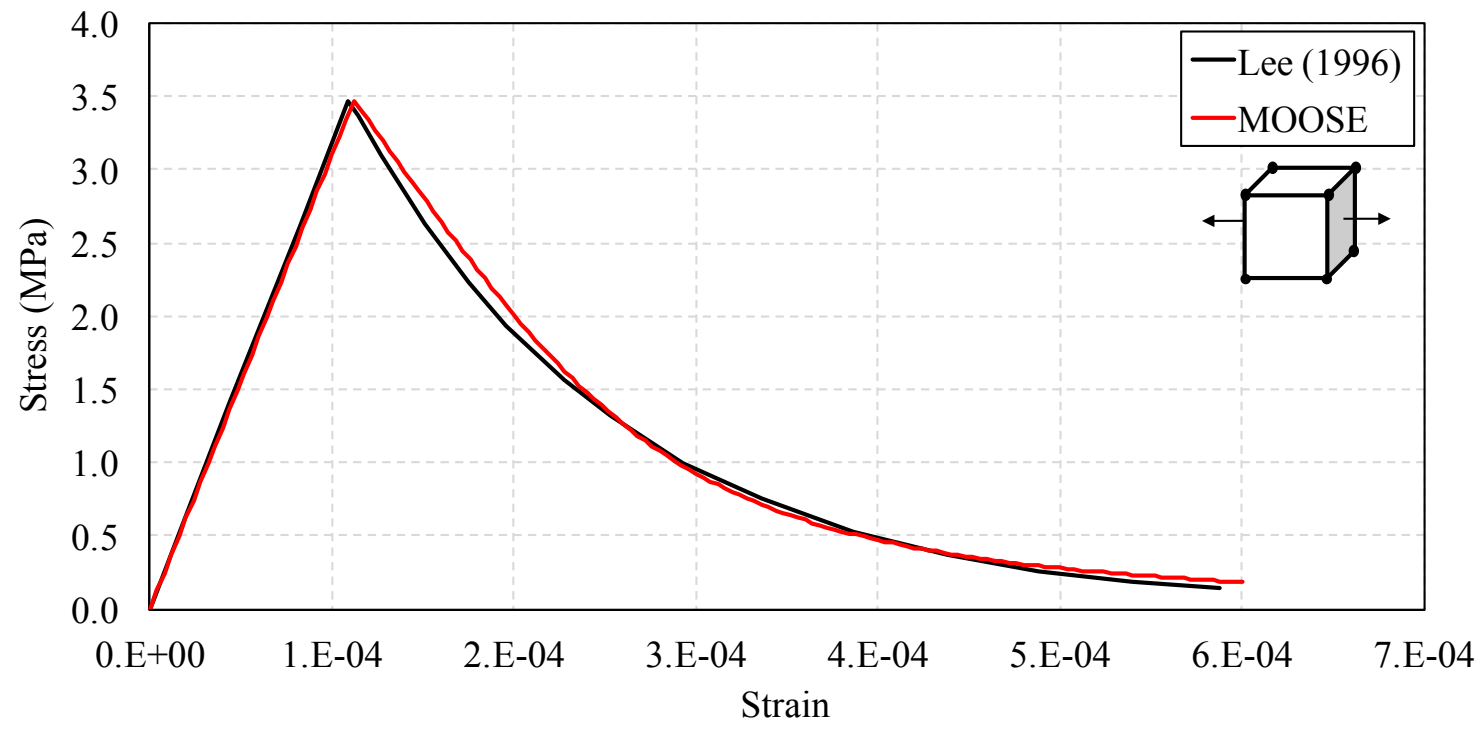

(a) 


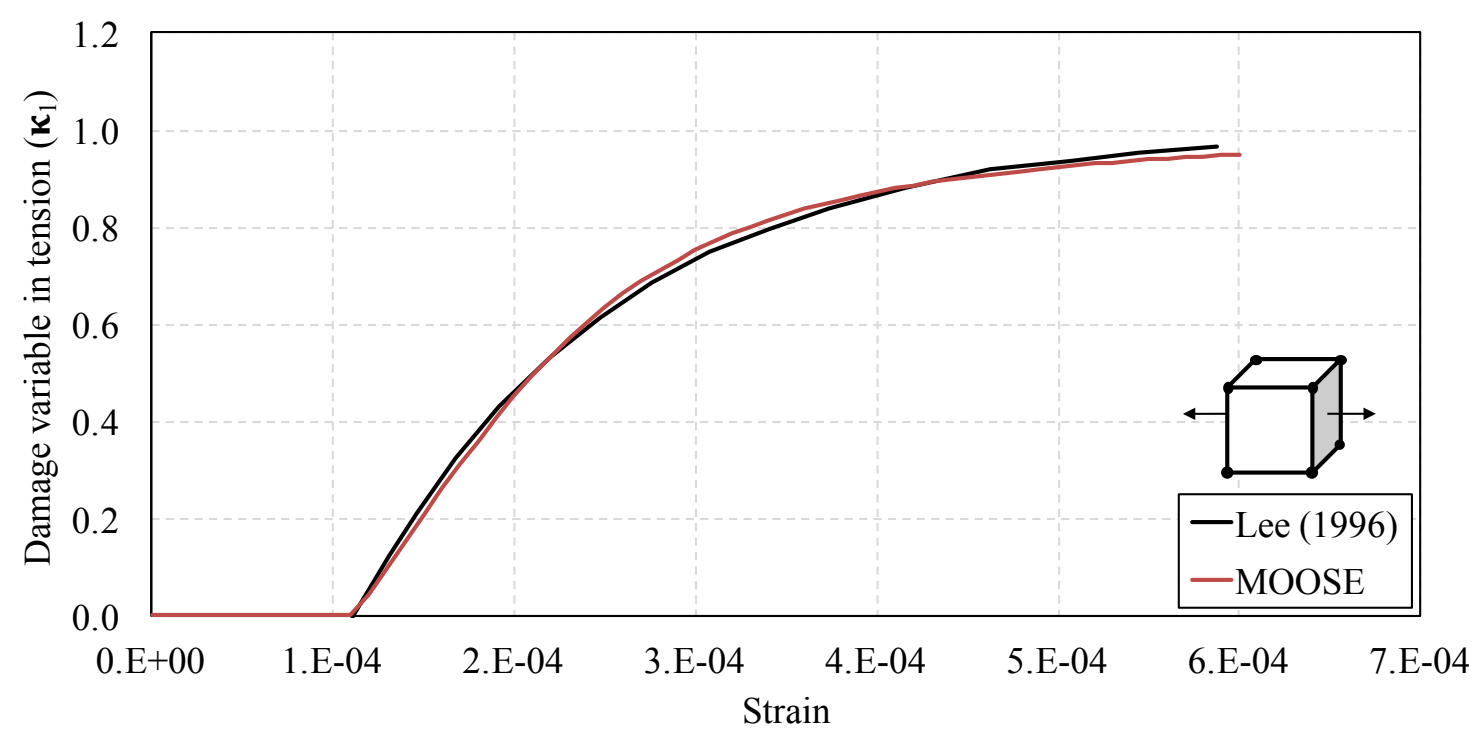

(b)

Figure 3: Comparison of results for (a)stress strain behavior (b) evolution of damage variables, in uniaxial tension

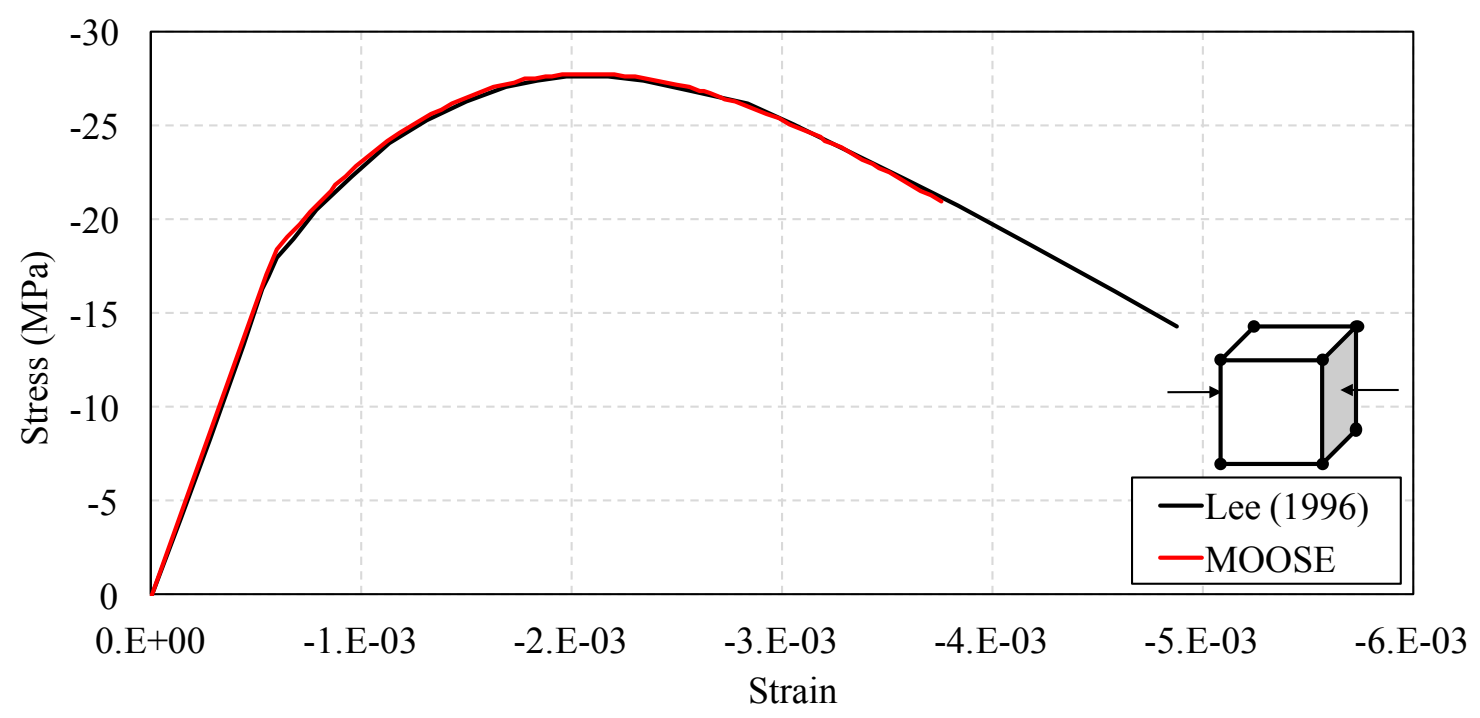

Figure 4: Comparison of results for uniaxial compressive loading 


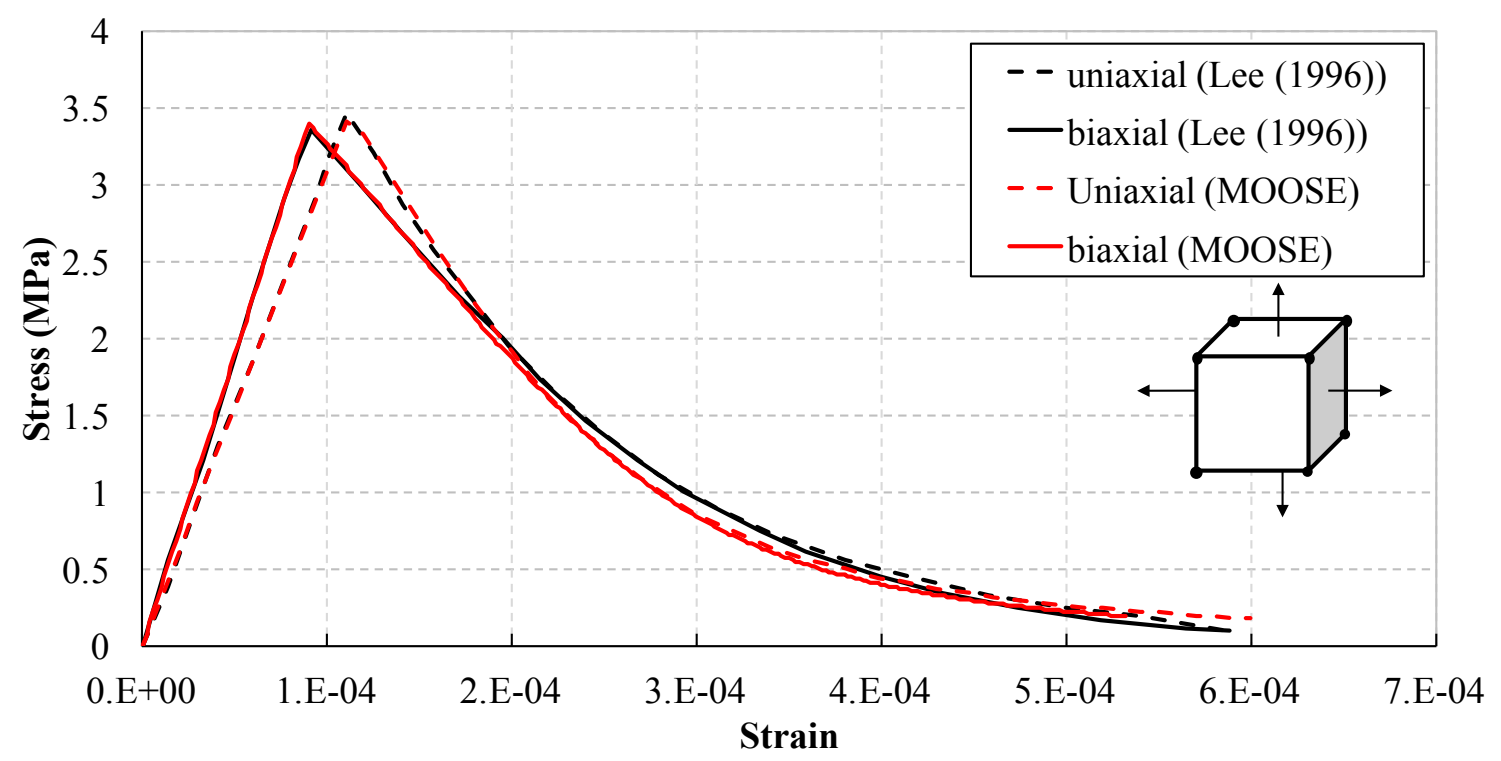

Figure 5: Comparison of results for biaxial tensile loading

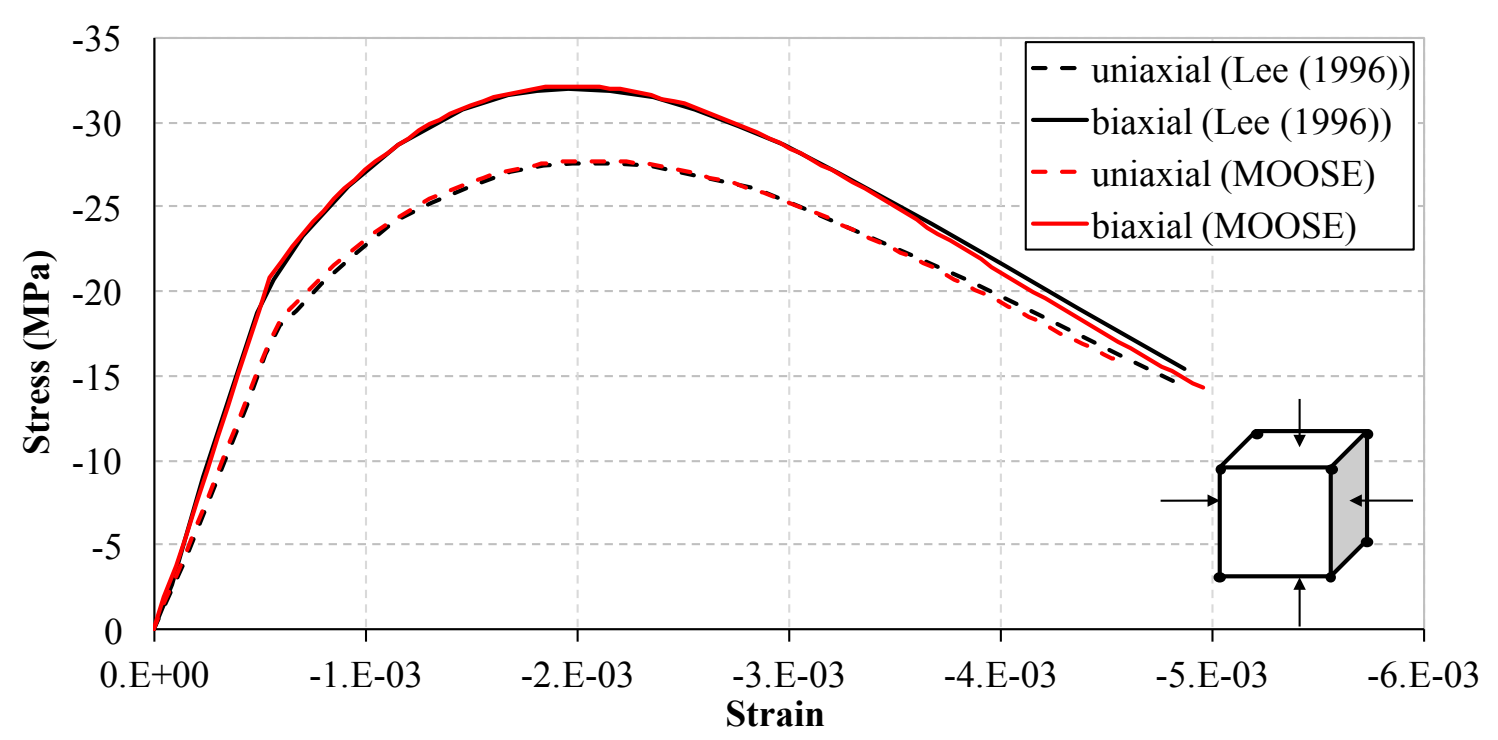

Figure 6: Comparison of results for biaxial compressive loading 


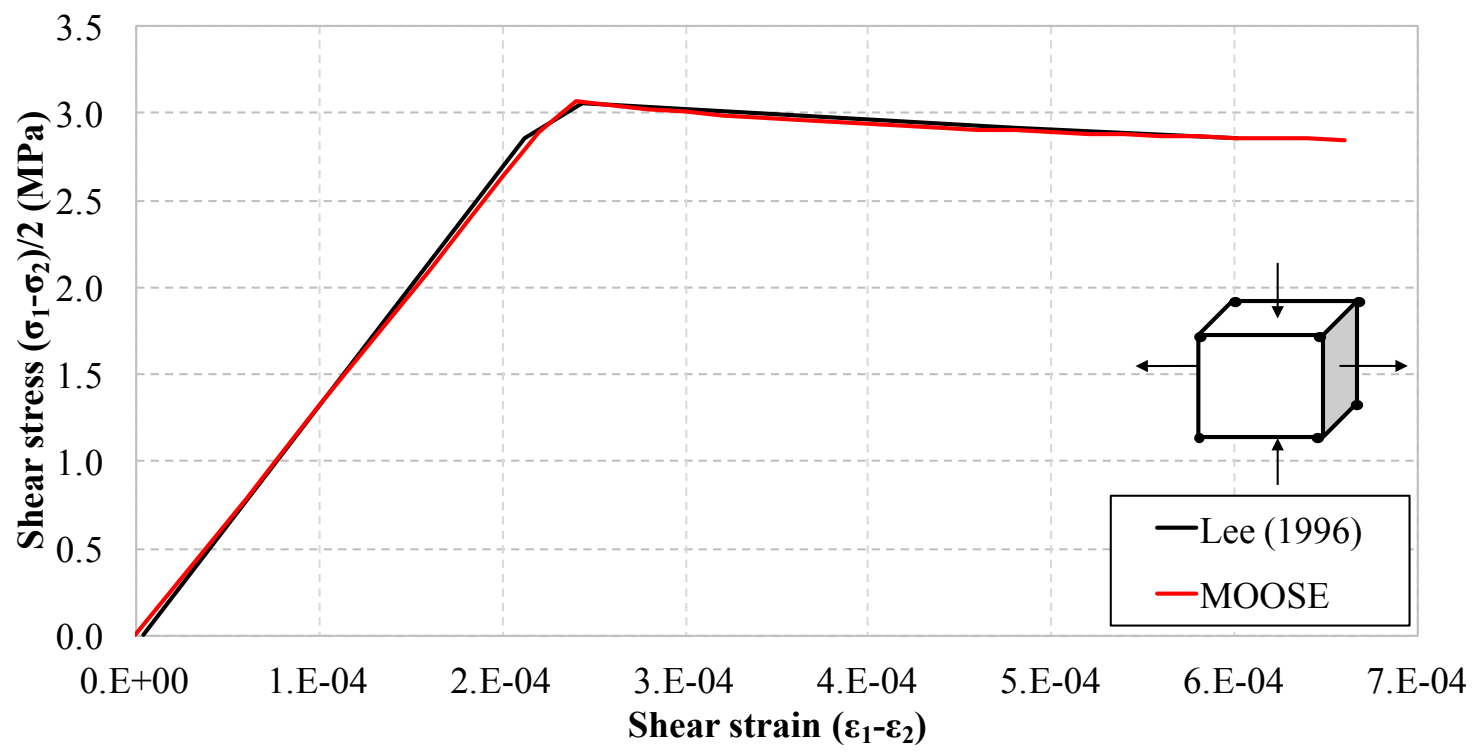

Figure 7: Comparison of results for shear loading

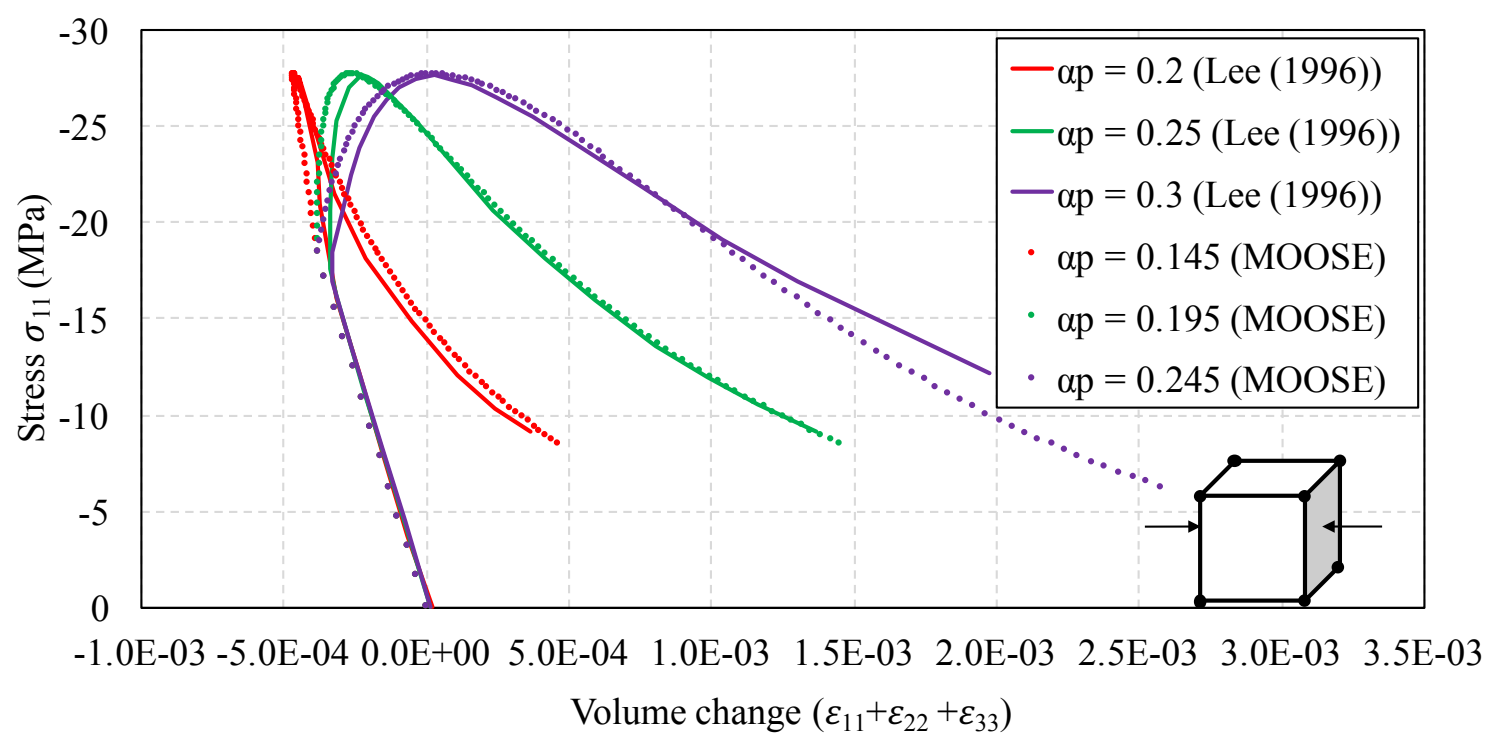

Figure 8: Comparison of results for dilatancy behavior in uniaxial compression

The implementation was also tested on multi-element models for uniaxial compression and split tension test of a concrete cylinder. The parameters used are same as those mentioned in Table 2 . Results of concrete cylinder are shown in Figure 9. The loading and boundary conditions for split tension test are provided in Figure 10. It was observed that the implementation works well in both strain hardening in compression and strain softening in tension. 


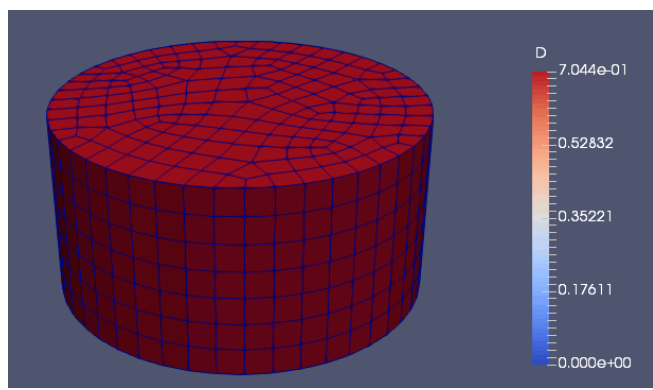

(a)

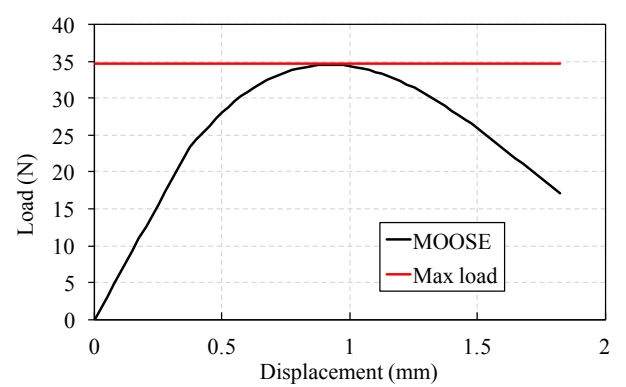

(b)

Figure 9: Concrete cylinder in uniaxial compression loading. (a) damage in element at maximum load, (b) load-displacement curve and comparison of maximum value with theoretically assumed value.

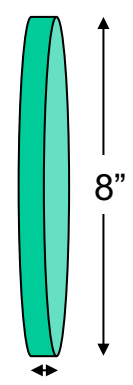

$0.1 "$

(a)

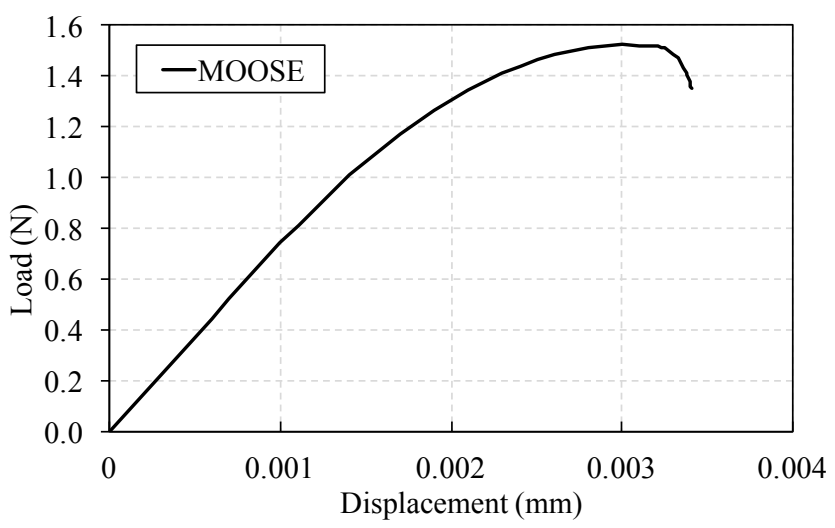

(d)

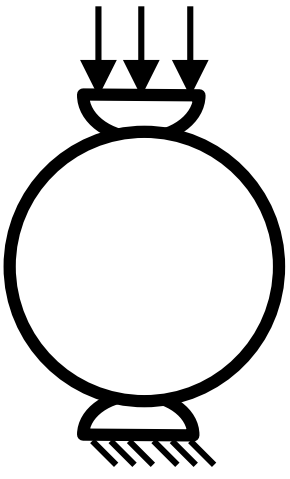

(b)

Figure 10: (a) Geometry of the specimen, (b) loading and boundary conditions, (c) mesh, (d) loaddisplacement curve.

\subsection{Shrinkage Strain Model}

The strain in the mechanical problem is calculated based on the assumption of a small strain, therefore if shrinkage effects are taken into account, the total strain tensor can be expressed as:

$$
\varepsilon_{i j}=\varepsilon_{i j}{ }^{e}+\varepsilon_{i j}{ }^{s h}
$$


where $\dot{\varepsilon}_{i j}$ is the total strain rate; $\dot{\varepsilon}_{i j}^{e}$ is the elastic strain rate; and, $\dot{\varepsilon}_{i j}^{e}$ is the rate of the drying shrinkage strain, which is the result of the movement of moisture in the concrete. The moisture movement is described by the pore relative humidity; thus, the drying shrinkage is related to the pore relative humidity. In this work, the relationship between the obtained moisture distribution and the drying shrinkage strain $\varepsilon_{s h}$ is used as follows:

$$
\varepsilon_{i j}^{s h}=\beta \Delta H \delta_{i j}
$$

where $\beta_{s h}$ is the shrinkage coefficent; $\dot{\varepsilon}_{s h}$ is the rate of the shrinkage strain; and, $\Delta H$ is the variation in the relative humidity. The shrinkage strain in the concrete is then calcluted using a multiphase composite model developed by Xi and Jennings (1997). This model is based on a three-phase, generalized, self-consistent model developed by Christensen (1979) for establishing elastic property. Based on the multiphase composite model, the shrinkage strain and the bulk modulus of concrete at the mesoscale level can be expressed as:

$$
\begin{gathered}
\varepsilon_{c o n c}^{s h}=\frac{K_{1} \varepsilon_{1}^{s h} g_{i}\left(3 K_{2}+4 G_{2}\right)+K_{2} \varepsilon_{2}^{s h}\left(1-g_{i}\right)\left(3 K_{1}+4 G_{2}\right)}{K_{2}\left(3 K_{1}+4 G_{2}\right)-4 g_{i} G_{2}\left(K_{2}-K_{1}\right)} \\
K_{c o n c}=\frac{K_{2}\left(3 K_{1}+4 G_{2}\right)-4 g_{i} G_{2}\left(K_{2}-K_{1}\right)}{\left(3 K_{1}+4 G_{2}\right)+3 g_{i}\left(K_{2}-K_{1}\right)}
\end{gathered}
$$

Where $\varepsilon_{1}^{s h}$ and $\varepsilon_{2}^{s h}, \mathrm{~K} 1$ and $\mathrm{K} 2$, and G1 and G2 are the shrinking, the bulk modulus, and the shear modulus for phase 1 and phase 2, respectively; and, gi is the aggregate volume fraction, and since shrinkage of the aggregates is too small and can be ignored, only the shrinkage of the cement paste would contribute to the shrinkage of the concrete:

$$
\varepsilon_{\text {conc }}^{s h}=\varepsilon_{2}^{s h}\left(1-\frac{g_{i}\left(K_{1} / K_{2}\right)}{1+\left(K_{1} / K_{2}-1\right) \frac{3+4 g_{i}\left(G_{2} / K_{2}\right)}{3+4\left(G_{2} / K_{2}\right)}}\right)
$$

\section{Multiscale modeling of chloride Ingress}

This section will present finite element model for simulating the effect of isotropic damage induced by drying shrinkage on the coupled moisture and chloride diffusion in concrete. The effect of the drying shrinkage damage on the chloride and moister diffusion coefficients is considered by using the concept of composite damage mechanic (Ababneh and Xi 2002), while the moisture capacity is evaluated by composite model for effective moisture capacity of a twophase composite ( $\mathrm{Xi}$ et al. 1994). The effects of shrinkage induced by drying are characterized through the framework of elastic damage mechanics. The objective of this model is to be able to predict the damage that may induced by shrinkage and also reveal the interactive effects between shrinkage and coupled chloride and that of moisture diffusion 


\subsection{Governing Differential Equations for Coupled Moisture and Chloride Diffusion}

\subsubsection{Chloride Diffusion}

The flux of chloride ions $J_{c l}$ into a unit area of saturated porous media based on Fick's first law can be expressed as:

$$
J=-D \nabla C
$$

where $J_{c 1}$ is the chloride ion flux, $D_{c l}$ is the chloride diffusion coefficient, and $C_{c l}$ is the free chloride concentration.

\subsubsection{Moisture Diffusion}

The moisture in concrete can be represented by pore relative humidity $(\mathrm{H})$ or by water content (w). In the present formulation, the moisture content is expressed by pore relative humidity, which is considered to be an indicator for combined water vapor and liquid water (Bazant and Najjar 1972). The flux of the moisture can be defined as a function of the gradient of the pore relative humidity:

$$
J_{H}=-D_{H} \nabla H
$$

in which $\mathrm{J}_{H}$ is the flux, $\mathrm{D}_{H}$ is the humidity diffusion coefficient, and $\mathrm{H}$ is the pore relative humidity.

\subsubsection{Coupled Moisture and Chloride Diffusion}

The transport processes of moisture and chloride ions in non-saturated concrete are considered to be two-way coupling processes between the two driving forces of moisture gradient and chloride concentration gradient (Ababneh and Xi 2003). Therefore, the equations of the chloride flux and moisture flux need to be modified to include the coupling terms as follows:

$$
\begin{gathered}
J_{C l}=-D_{C l} \nabla C_{C l}-D_{C l-H} \nabla H \\
J_{H}=-D_{H-C l} \nabla C_{C l}-D_{H-H} \nabla H
\end{gathered}
$$

where $D_{c l-c l}$ is the chloride diffusion coefficient $\left(\mathrm{cm}^{2} /\right.$ day); $C_{c l}$ is the free chloride concentration (in gram of free chloride per gram of concrete, $\mathrm{g} / \mathrm{g}$ ) $; D_{H-H}$ is the humidity diffusion coefficient ( $\mathrm{cm}^{2} /$ day); $H$ is the pore relative humidity; $D_{c l-H}$ is the coupling parameter - which represents the coupling effect of moisture diffusion on chloride penetration; and, $D_{H-c l}$ is the coupling parameter- which represents the coupling effect of chloride ions on moisture diffusion. The governing equations for coupled chloride and moisture diffusion can be written using the mass conservation equation and the flux equations as follows: 


$$
\begin{gathered}
\frac{\partial C_{t}}{\partial C_{f}} \frac{\partial C_{f}}{\partial t}=\operatorname{div}\left[D_{c l-c l} \nabla C_{f}\right]+\operatorname{div}\left[D_{C l-H} \nabla H\right] \\
\frac{\partial w}{\partial H} \frac{\partial H}{\partial t}=\operatorname{div}\left[D_{H-c l} \nabla C_{f}\right]+\operatorname{div}\left[D_{H-H} \nabla H\right]
\end{gathered}
$$

in which $\partial C_{f} / \partial C_{t}$ is the chloride binding capacity and is the moisture binding capacity; $C_{t}$ is the total chloride concentration (in gram of free chloride per gram of concrete, $\mathrm{g} / \mathrm{g}$ ), and $w$ is the moisture content in concrete.

\subsection{Multiscale material models}

In the following, detailed description of the material models used to determine the material parameters involved in the governing equations of the transport proses in the concrete. These parameters include: the binding capacities, the diffusion coefficients, and the coupling parameter

\subsubsection{Moisture capacity}

The moisture capacity of the concrete can be determined using the multiscale and multiphase model developed by Xi (1995). In this model, the concrete is considered as two-phase material, in which phase one is representing the aggregates (inclusions), and phase two is representing the cement paste (Matrix). The moisture capacity of the concrete is then defined as the average of the moisture capacities of the two phases as follow:

$$
\frac{\partial w}{\partial H}=f_{a g g}\left(\frac{\partial w}{\partial H}\right)_{a g g}+f_{c p}\left(\frac{\partial w}{\partial H}\right)_{c p}
$$

Here, $f_{c p}$ and $f_{a g g}$ are the weight percentages of the cement paste and the aggregate, and $(\partial \mathrm{w} / \partial H)_{a g g}$ and $(\partial \mathrm{w} / \partial H)_{c p}$ are the moisture capacities of the aggregate and the cement paste. The moisture capacity of the cement paste is defined as the derivative of the adsorption isotherm of the porous media (the relationship between moisture content and relative humidity at a constant temperature in the porous media) where the adsorption isotherm for a porous media can be described by BSB theory (Brunauer et al. 1969):

$$
w=\frac{C k V_{m} H}{(1-k H)[1+(C-1) k H]}
$$

From Eq (54) the moisture capacity can be expressed as:

$$
\frac{\partial w}{\partial H}=\frac{C \quad k \quad V_{m}+w k[1+(C-1) k H]-w k[(C-1)(1-k H)]}{(1-k H)[1+(C-1) k H]}
$$

Where, $H$ is the relative humidity, parameters $V_{m}, C$, and $k$ are the monolayer capacity (mass of adsorbate required to cover the adsorbent with a single molecular layer), the net heat absorption, 
and the degree of saturation of the pores, respectively. The parameter $V_{m}$ can be determined using the Xi et al. (1994b, 1994a) model as follows:

$$
V_{m}=\left(0.068-\frac{0.22}{t}\right)\left(0.85+0.45 \frac{w}{c}\right) V_{c t} \quad t>5 \text { days }, \quad 0.3<\frac{w}{c}<0.7
$$

In the above: $\mathrm{t}$ is the time in days; $\mathrm{w} / \mathrm{c}$ is the water-cement ratio; and, $V_{c t}$ is a material constant, which takes the value of $0.9,1,0.85$ and 0.6 for cement types I, II, III, and IV, respectively. The parameter $\mathrm{C}$ is expressed as:

$$
C=\exp \left(\frac{C_{o}}{T}\right)
$$

where $\mathrm{T}$ is the temperature in (Kelvin) and $C_{0}=855$. The parameter $\mathrm{k}$ is expressed as:

$$
k=\frac{(1-1 / n) C-1}{C-1}
$$

and here $\mathrm{n}$ represents the number of the adsorbed layers in the pore, which is defined as a function of time (days), water-cement ratio, and cement type:

$$
n=\left(2.5+\frac{15}{t}\right)\left(0.33+2.2 \frac{w}{c}\right) N_{c t} \quad t>5 \text { days }, \quad 0.3<\frac{w}{c}<0.7
$$

where $N_{c t}=1.1,1.0,1.15$, and 1.5 for cement types I, II, III, and IV, respectively. The moisture capacity of aggregates can be evaluated by using Eq (55). However, parameters $V_{m}$ and $\mathrm{n}$ are determined using the following formulas (Xi 1995):

$$
\begin{gathered}
V_{m}=0.0647 V_{a g g} \\
n=4.063 n_{a g g}
\end{gathered}
$$

where $\mathrm{V}_{\mathrm{agg}}$ and $\mathrm{n}_{\mathrm{agg}}$ are constants dependent upon the aggregate structures. For dense aggregates, such as crushed limestone, river gravel, dolomite, and granite, the value of the $\mathrm{V}_{\text {agg }}$ ranges from 0.05 to 0.1 and the value of $n_{\text {agg }}$ ranges from 1.0 to 1.5. For lightweight aggregates, the value of the $\mathrm{V}_{\text {agg }}$ ranges from 0.01 to 0.04 and the value of $\mathrm{n}_{\text {agg }}$ ranges from 1.7 to 2.0.

\subsubsection{Humidity diffusion coefficient.}

Based on the composite theory of Christensen (1979), the effective humidity diffusion coefficient of concrete can be expressed as a function of the diffusion coefficient of cement paste and the diffusion coefficient of aggregate:

$$
D_{H}=D_{H c p}\left(1+\frac{g_{i}}{\left[1-g_{i}\right] / 3+1 /\left[\left(D_{\text {Hagg }} / D_{H c p}\right)-1\right]}\right)
$$

where $\mathrm{D}_{\text {Hср }}$ is the humidity diffusion coefficient of the cement paste; $g_{i}$ is the aggregate volume fraction; and, $\mathrm{D}_{\mathrm{Hagg}}$ is the humidity diffusion coefficient of the aggregate. Because the pores in 
the aggregate are discontinuous, the value of the humidity diffusion coefficient of the aggregate is too small to compare with the value of the humidity diffusion coefficient of cement paste.

Thus, $\mathrm{D}_{\text {Hagg }}$ in Eq.(20) can be ignored. The humidity diffusion coefficient of the cement paste can be calculated by using the empirical model developed by Xi et al. (1994) as follows:

$$
D_{H c p}=\alpha_{h}+\beta_{h}\left[1-\exp \left(-\ln 2 * 10^{\gamma_{h}(H-1)}\right)\right]
$$

where the coefficients $\alpha_{\mathrm{h}}, \beta_{\mathrm{h}}$, and $\gamma_{\mathrm{h}}$ are calculated using the following formulas:

$$
\begin{aligned}
& \alpha_{h}=1.05-3.8\left(\frac{w}{c}\right)+3.56\left(\frac{w}{c}\right)^{2} \\
& \beta_{h}=-14.4-50.4\left(\frac{w}{c}\right)-41.8\left(\frac{w}{c}\right)^{2} \\
& \gamma_{h}=31.3-136\left(\frac{w}{c}\right)+162\left(\frac{w}{c}\right)^{2}
\end{aligned}
$$

\subsubsection{Chloride binding capacity}

The chloride binding capacity of concrete is defined as the ratio between the rate of change in the free chloride concentration and the rate of change in the total chloride concentration:

$$
\frac{d C_{f}}{d C_{t}}=\frac{1}{\frac{d C_{t}}{d C_{f}}}=\frac{1}{\frac{d\left(C_{f}+C_{b}\right)}{d C_{f}}}=\frac{1}{1+\frac{d C_{b}}{d C_{f}}}
$$

In this study, an empirical model developed by Xi and Bazant (1999) based on the Ferundlich isotherm, is used:

$$
\frac{d C_{f}}{d C_{t}}=\frac{1}{1+\frac{A 10^{B} \beta_{C-S-H}}{35450 \beta_{\text {sol }}}\left(\frac{C_{f}}{35.45 \beta_{\text {sol }}}\right)^{A-1}}
$$

where, $\mathrm{A}$ and $\mathrm{B}$ are material constants related to chloride adsorption that take the values 0.3788 and 1.14, respectively (Luping and Nilsson 1993). $\beta \mathrm{C}-\mathrm{S}-\mathrm{H}$ is the ratio between the weight of $\mathrm{C}$ $\mathrm{S}-\mathrm{H}$ gel and the weight of concrete $(\mathrm{g} / \mathrm{g}),(\mathrm{Xi}$ and Bazant 1999), as follows:

$$
\beta_{C-S-H}=\frac{w_{C-S-H}}{w_{\text {total }}}
$$

In the following, $\beta_{\text {sol }}$ is the ratio between the volume of the pore solution and the weight of the concrete (L/g)(Xi and Bazant 1999): 


$$
\beta_{\text {sol }}=\frac{V_{\text {sol }}}{w_{\text {conc }}}=\frac{w_{\text {sol }}}{\rho_{\text {sol }} w_{\text {conc }}}=\frac{n(H, T)}{\rho_{\text {sol }}}
$$

where, $w_{\text {conc }}$ is the weight of concrete; $w_{\text {sol }}$ and $V_{\text {sol }}$ are respectively the weight and the volume of the pore solution; and, $\varrho_{\text {sol }}$ is the density of the pore solution $(\mathrm{g} / \mathrm{L})$, which is dependent on the chloride concentration. The term $w_{\text {sol }} / w_{\text {conc }}$ is the chloride adsorption isotherm, which is defined as a function of the temperature $T$ and relative humidity $H$ of the concrete. Since there is no test data available on the chloride adsorption isotherm, the water adsorption isotherm is utilized. The expression of the water adsorption isotherm is given by averaging the adsorption isotherm of the aggregate and that of the cement paste as follows:

$$
n(H, T)=f_{c p} n_{c p}(H, T)+f_{g} n_{g}(H, T)
$$

Here, $f_{a g g}$ and $f_{c p}$ are the weight percentages of the aggregate and the cement paste; $n_{a g g}(H, T)$ and $n_{c p}(H, T)$ are the water adsorption isotherms of the aggregate and the cement paste, respectively. Eq. (68) is valid only when the free chloride concentration is greater than $0.01 \mathrm{~mol} / 1$. The limitation of Eq. (68) is that, when the free chloride concentration $C_{f}$ is zero, the value of $d C_{f}$ $/ d C_{t}$ will be equal to zero which will cause $d C_{f} / d t$ to also be equal to zero. All this means that $C_{f}$ will remain constant at all-time steps and the diffusion process will not begin. In this work, the Langmuir isotherm is used when the value of free chloride concentration $C_{f}$ is less than 0.05 $\mathrm{mol} / \mathrm{l}$. The Langmuir isotherm is expressed as:

$$
\frac{1}{C_{b}^{\prime}}=\frac{1}{k^{\prime} C_{b m}} \frac{1}{C_{f}^{\prime}}+\frac{1}{C_{b m}}
$$

where, $C_{f}$ ' and $C_{b}$ ' are the free and the bound chloride concentrations; $C_{b m}$ is the bounded chloride concentration at saturated monolayer adsorption; and, $k^{\prime}$ is an adsorption constant (Luping and Nilsson 1993). The value of $k^{\prime}$ and $C_{b m}$ can be obtained by curve fitting the adsorption test data. For Eq.(72), $C_{f}{ }^{\prime}$ and $C_{b}$ ' are represented as moles per liter of pore solution $(\mathrm{mol} / \mathrm{L})$ and milligrams of bounded chloride per gram of C-S-H gel $(\mathrm{mg} / \mathrm{g})$, respectively. In order to use consistent units, the units of $C f^{\prime}$ and $C b^{\prime}$ are correlated using the $\beta$ sol and $\beta C-S-H$ as follows:

$$
\begin{gathered}
C_{f}^{\prime}=\frac{C_{f}}{35.45 \beta_{\text {sol }}} \\
C_{b}^{\prime}=\frac{1000 C_{b}}{\beta_{C-S-H}}
\end{gathered}
$$

Substituting Eqs. (73) and (74) Eq. (72) yields

And, rearranging Eq. (75),

$$
\frac{1}{C_{b}}=\frac{1000}{\beta_{C-S-H}}\left[\frac{35.45 \beta_{s o l}}{k^{\prime} C_{b m}} \frac{1}{C_{f}}+\frac{1}{C_{b m}}\right]
$$

where,

$$
C_{b}=\frac{1}{\beta+\frac{1}{\alpha C_{f}}}
$$




$$
\begin{gathered}
\alpha=\frac{k^{\prime} C_{b m} \beta_{C-S-H}}{35450 \beta_{s o l}} \\
\beta=\frac{1000}{\beta_{C-S-H} C_{b m}}
\end{gathered}
$$

Taking the derivative of Eq. (75) with respect to $\mathrm{C}_{f}$, we obtain:

$$
\frac{d C_{b}}{d C_{f}}=\frac{1}{\alpha\left(C_{f}\right)^{2}\left(\beta+\frac{1}{\alpha C_{f}}\right)^{2}}
$$

Substituting Eq. (79) into Eq. (67), we obtain the binding capacity based on Langmuir isotherm:

$$
\frac{d C_{f}}{d C_{t}}=\frac{1}{1+\frac{d C_{b}}{d C_{f}}}=\frac{1}{1+\frac{1}{\alpha\left(\beta C_{f}+\frac{1}{\alpha}\right)^{2}}}
$$

\subsubsection{Chloride diffusion coefficient $D_{c l}$}

The chloride diffusion coefficient of concrete $\mathrm{D}_{c l}$ can be determined by using the multifactor method as follows

$$
D_{c l}=f_{1}\left(t_{o}, \frac{w}{c}\right) f_{2}\left(g_{i}\right) f_{3}(H) f_{4}(T) f_{5}\left(C_{f}\right)
$$

where the first factor, $f_{l}\left(t_{o}, w / c\right)$, accounts for the effects of curing time and water-cement ratio on the chloride diffusion coefficient. Xi and Bazant (1999) proposed the model for this factor. It is represented as follows:

$$
f_{1}\left(\frac{w}{c}, t_{o}\right)=\frac{28-t_{o}}{62500}+\left(\frac{1}{4}+\frac{\left(28-t_{o}\right)}{300}\right)\left(\frac{w}{c}\right)^{6.55}
$$

The second factor, $f 2\left(g_{i}\right)$, accounts for the effect of the composite action on the cement paste and aggregate. The expression for this factor is similar to that expressed in Eq.(62) (Christensen 1979):

$$
f_{2}\left(g_{i}\right)=D_{c p}\left(1+\frac{g_{i}}{\left[1-g_{i}\right] / 3+1 /\left[\left(D_{a g g} / D_{c p}\right)-1\right]}\right)
$$

where $g_{i}$ is the aggregate volume fraction; and, $D_{\mathrm{cp}}$ and $D_{a g g}$ are the chloride diffusion coefficients of cement paste and aggregate, respectively. The value of $D_{c p}$ and $D_{a g g}$ can be determined by using the model for porous media created by Martys and Torquato (1994), and represented as: 


$$
D=\frac{2\left(1-\left(V_{p}-V_{p}^{c}\right)\right)}{S^{2}}\left(V_{p}-V_{p}^{c}\right)^{4.2}
$$

In the above, $V_{p}, V_{p}{ }^{c}$, and $S$ are the porosity, the critical porosity, and the surface area, respectively. When Eq.(84) is used to determine the value of the chloride diffusion coefficient of the cement paste, the porosity of the cement paste $V_{p}$ is estimated by using the value of the adsorption isotherm at $\mathrm{H}=100 \%$, which is defined as the water content at saturation. The critical porosity of the cement paste $V_{p}^{c}$ is assumed to be 3\% according to Martys and Torquato (1994). The surface area of the cement paste is related to the monolayer capacity and it can be determined using Eq.(56).

The third factor, $f 3(H)$, accounts for the effect of the relative humidity. Bazant and Najjar (1972) proposed a model that accounts for the influence of the relative humidity on moisture diffusion and the same model can be applied in this case:

$$
f_{3}(H)=\left(1+\frac{(1-H)^{4}}{\left(1-H_{C}\right)^{4}}\right)^{-1}
$$

Here, $H$ is the relative humidity and $H_{c}$ is the critical humidity level at which the diffusion coefficient drops halfway between its maximum and minimum values $\left(H_{c}=0.75\right)$. The fourth factor $f 4(T)$, accounts for the effect of temperature. Arrhenius' law is used to account for the temperature effect on the chloride diffusion coefficient, as shown below:

$$
f_{4}(T)=\exp \left[\frac{U}{R}\left(\frac{1}{T_{o}}-\frac{1}{T}\right)\right]
$$

Here, $R$ is the gas constant $8.314 \mathrm{~J} \mathrm{~mol}^{-1} \mathrm{~K}^{-1} ; T_{o}$ and $T$ are the reference and the current temperatures in Kelvin, respectively, $\left(\mathrm{T}_{\mathrm{o}}=296 \mathrm{~K}^{\circ}\right)$; and, $U$ is the activation energy, which depends on cement type and water-cement ratio (Page et al. 1981). Table 3 shows various watercement ratios and some test results for various values of the activation energy $U$.

Table 3: Activation Energies for Various Cement Pastes

\begin{tabular}{lc}
\hline W/C & U for Ordinary Portland Cement $(\mathrm{KJ} / \mathrm{mol})$ \\
\hline 0.4 & $41.8 \pm 4.0$ \\
\hline 0.5 & $44.6 \pm 4.3$ \\
\hline 0.6 & $32.0 \pm 2.4$ \\
\hline
\end{tabular}

The fifth factor $f_{5}\left(C_{f}\right)$ accounts for the effect of free chloride concentration on the chloride diffusion coefficient, which can be expressed as follows:

$$
f_{5}\left(C_{f}\right)=1-k_{\text {ion }}\left(C_{f}\right)^{m}
$$

where, $k_{\text {ion }}=8.333$ and $\mathrm{m}=0.5(\mathrm{Xi}$ and Bazant 1999). 


\subsubsection{Coupling parameters.}

Coupling parameters $\left(D_{i-H}\right)$

A material model for the coupling parameter $\left(D_{i-H}\right)$ developed based on an experimental study on the effect of $\mathrm{w} / \mathrm{c}$ ratio and aggregate volume fraction on chloride penetration in non-saturated concrete was used in this work as shown below:

$$
D_{i-H}=3.27 \times 10^{-7} \cdot\left(1.42 \times 10^{13}\right)^{w / c} \cdot \frac{c_{i}}{t} \cdot D_{m}\left(1+\frac{g_{i}}{\left[1-g_{i}\right] / 3+1 /\left[\left(D_{i} / D_{m}\right)-1\right]}\right)
$$

Where $C_{i}$ is the concentration, $g_{i}$ is the aggregate volume fraction, the value of $D_{i}$ and $D_{m}$ are determined based on curve fitting of the test data of 0.00496 and $1.84 \mathrm{E}-06$, respectively. Coupling parameters $\left(D_{H-i}\right)$

Currently, the value of this used in the finite element program are based on the studies of Ababneh and Xi (2003) and Homan et al. (2016). These researchers concluded that the values of coupling parameters are not constant but rather strongly dependent upon the concentration of free chloride and therefore can be expressed in terms of free chloride concentration. The relationship between the free chloride concentration and the coupling parameters $D_{c l-H}$ and $D_{H-c l}$ are determined by curve fitting the test data to 0.19 and 0.52 , respectively. However, for the effect of multi-species on the moisture diffusion cannot be treated in the same way. This is because the effect of the diffusion of a specific ion on the moisture movement varies with the diffusion rate of the ion. Therefore, the coupling parameter, $D_{H-i}$ of each species can be estimated by the ratio between a specific ionic species and the chloride ion as shown

$$
\frac{D_{i}}{D_{c l}}=\frac{D_{H-C i}}{D_{H-c l}}=\frac{\delta_{i} C_{i}}{\delta C_{c l}}
$$

\subsection{The Effect of Drying Shrinkage on Material Parameters}

\subsubsection{Moisture capacity}

A composite model for effective moisture capacity of two-phase composite material developed by Xi (1995)was used in this study. The model was developed using the extreme energy principle method utilized by Rosen and Hashin (1970) to evaluate the effect of thermal strain on the heat capacity of two-phase composite material. In this model, (Rosen and Hashin 1970) proposed that effective heat capacities could be expressed as a function of the volumetric average of the heat capacities of the constituent phases, the thermal expansion, and the bulk modulus of the material. Similarly, the effective moisture capacity can be represented by two terms. The first term is the volumetric average of the moisture capacities of the constituent phases, and the second is the coupling effect, which is defined as a function of the bulk modulus, the shrinkage coefficients of the constituents, and the volume fraction of the inclusion. The effective moisture capacity can be expressed as follows: 


$$
\frac{\partial w}{\partial H}=\left(\frac{\partial w}{\partial H}\right)_{\text {avg }}+\psi\left[9 \frac{H^{0}}{R T^{0}}+\left(\frac{\beta_{i}-\beta_{m}}{\frac{1}{k_{m}}-\frac{1}{K_{i}}}\right)^{2}\left[\left(\frac{\overline{1}}{K}\right) \frac{1}{K_{c o n c}}\right]\right]
$$

with

$$
\left(\frac{\overline{1}}{K}\right)=\frac{g_{m}}{K_{m}}+\frac{g_{i}}{K_{i}} \quad \text { and } \quad K_{\text {conc }}=K_{m}+\frac{g_{i}\left(K_{i}-K_{m}\right)}{1+g_{m}\left[\left(k_{i}-k_{m}\right) /\left(k_{m}+4 G_{m} / 3\right)\right]}
$$

where $(d w / d H)_{\text {avg }}$ is the volumetric average of moisture capacities of cement paste and aggregate without considering the effect of shrinkage, which can be determined based on BET adsorption isotherm; $\Psi$ is a factor used to convert units from $\mathrm{mole} / \mathrm{m}^{3}$ to gram $/ \mathrm{gram}$; and, $R$ is the gas constant in Joules/mole/K. The reference temperature and reference humidity are $\mathrm{T}^{\circ}$ and $\mathrm{H}^{\circ}$ (in Kelvin); $\beta_{i}$ and $\beta_{m}$ are the shrinkage coefficients for the aggregate and the cement paste; and, $G_{m}$ is the shear modulus of the cement paste. $K_{m}, K_{i}$, and $K_{\text {conc }}$ are the bulk modulus of cement paste, aggregate, and concrete, respectively. And, here $g i$ and $g m$ are the volume fraction of the aggregate and the cement paste.

\subsubsection{Diffusion coefficient.}

This study used a composite damaged model for diffusivity of distressed materials (Ababneh and $\mathrm{Xi}$ 2002). The model defines the distressed material as a two-phase composite material with intact material as phase one and distressed material as phase two. The effective diffusivity of the composite material can be characterized by using the research of Christensen (1979) who developed a model for determining the conductivity of the spherical spatial distribution of the constituent phases:

$$
D_{H}(H, w)=D_{2}\left[1+\frac{w}{(1-w) / 3+1 /\left[\left(D_{1} / D_{2}\right)-1\right]}\right]
$$

where $D_{1}$ is the (humidity/chloride) diffusivity of undamaged concrete; $D_{2}$ is the diffusivity of (humidity/chloride) in damaged concrete; and, $w$ is the scalar damage parameter, which will be determined based on the combined plasticity and damage theory. More detail will be provided in the following section.

Numerical Example:

The set of partial differential equations describing the effect of drying shrinkage-induced damage on coupled moisture and chloride diffusion into concrete were solved numerically using finite element method. The governing equations have been implemented in a 2D Finite element model using the Matlab environment. The numerical models have been tested for the selected example. In this example, two-dimensional concrete slab with a depth of $20 \mathrm{~cm}$ and a width of $30 \mathrm{~cm}$ was analyzed, as shown in Figure 11. The slab had an aggregate volume fraction of 0.65 and a watercement ratio of 0.55 . The material properties for the slab were as follows: $E o=31000 \mathrm{MPa}, n u=$ $0.18, A t=0.87, A c=0.65, B t=20000, B c=2150, k o=0.0001, f t=3.48 \mathrm{MPa}$, and $f o=-27.6$ $\mathrm{MPa}$. The initial relative humidity inside the slab was $50 \%$. The top surface of the slab was 
subjected to periodical wetting-drying cycles with a wetting duration of 10 days using a 5\% (by weight) sodium chloride solution, and a drying duration of 20 days. The assumption of plane strain was carried out during the analysis of the mechanical problem.

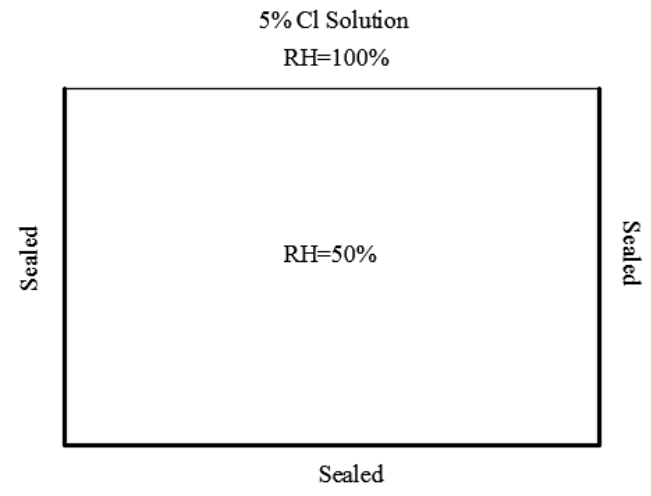

Transport Model

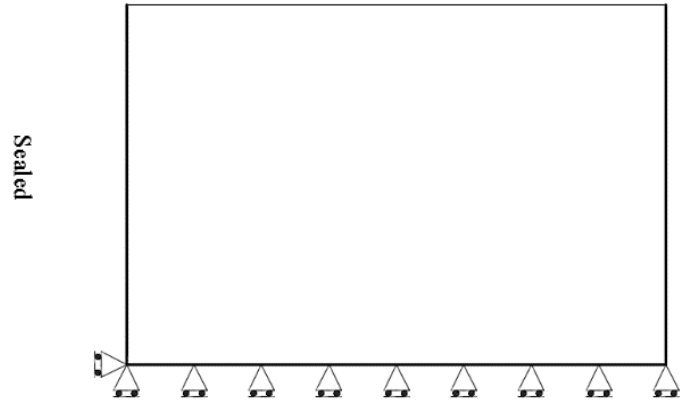

Mechanical Model

Figure 11: Mechanical and transport models for the slab

The effect of drying-shrinkage damage on the chloride ion diffusion and relative humidity are shown in Figures 12,13. These figures show the analysis of the slab under two circumstances. In the first scenario, the slab was subjected to periodical wetting-drying cycles without considering any evolution in damage. In the second scenario, the slab was subjected to the same periodic wetting-drying cycles with the evolution in damage noted. As shown in Figure 12, the chloride ions diffuse at a faster rate when the damage is considered in the analysis. This indicates that damage accelerates the chloride diffusion process.

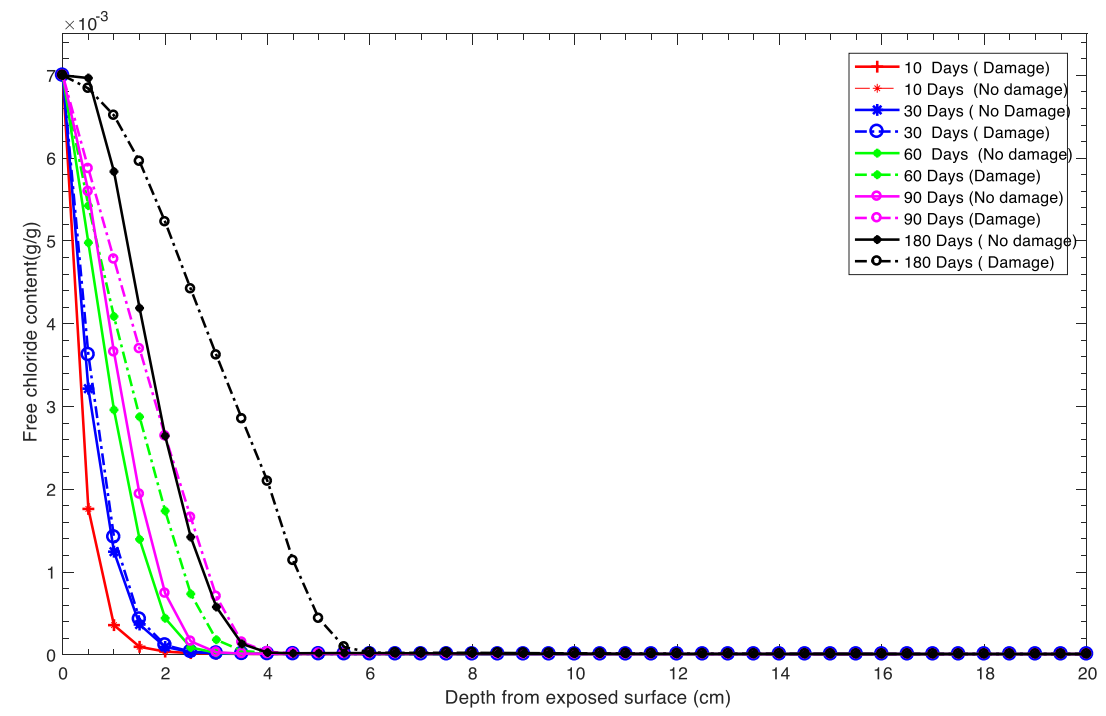

Figure 12: The effect of drying-shrinkage damage on chloride ion penetration 


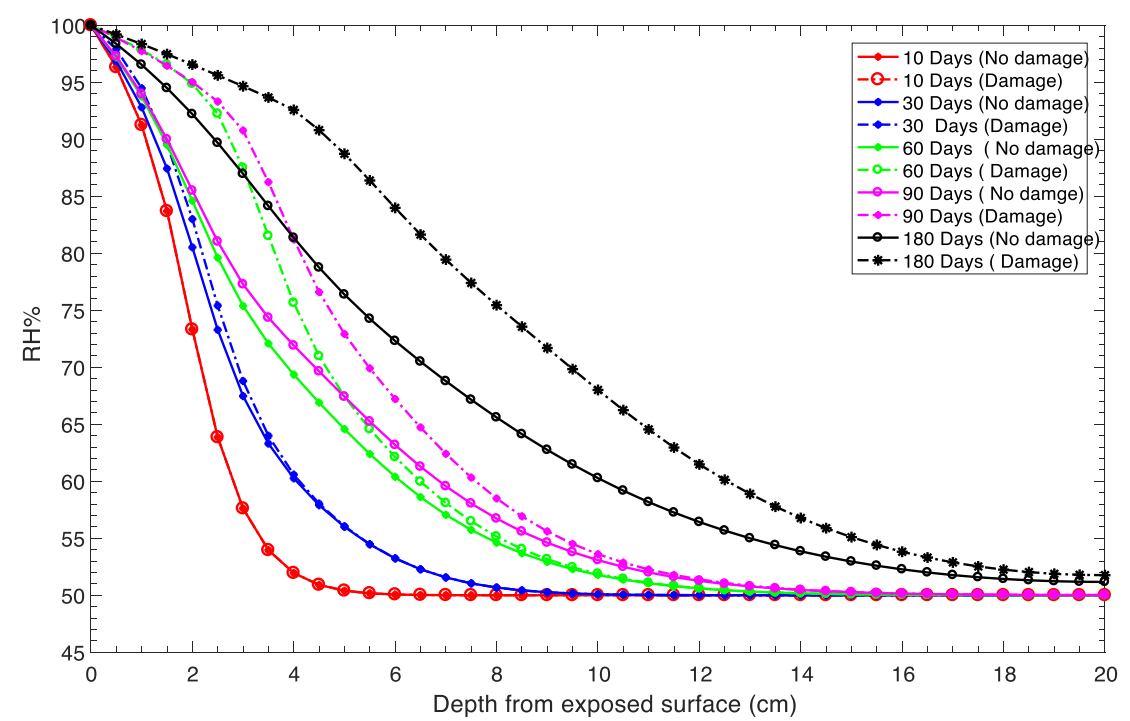

Figure 13. The effect of drying-shrinking damage on relative humidity.

\subsection{Modeling the Effect of Shrinkage-Induced Damage on Multi- Species Diffusion into Concrete}

As can be seen in the previous section, several material parameters affecting the penetration rate of chloride ions are taken into account when calculating the chloride diffusion coefficient and chloride binding capacity. The numerical model was developed using Fick's laws of diffusion, which is based on the assumption that the ions are considered to be as an uncharged particle. Thus, the electrical interactions between the chloride ion and other ions that may be present in the solution is not considered even though it is an experimental fact. When solution such as magnesium chloride $(\mathrm{MgCl} 2)$, calcium chloride $(\mathrm{CaCl} 2)$, and sodium chloride $(\mathrm{NaCl})$ penetrate into concrete, chloride is not the only ion type diffusing in the concrete; other ions from solution diffuse in the concrete simultaneously. In addition to these other ions, different ions that exist in the concrete pore solution such as $\mathrm{Na}+, \mathrm{K}+$, and $\mathrm{OH}-$, must be considered as well.

Based on the literature review and other research, a preliminary framework for modeling the shrinkage damage on multi-species diffusion in concrete was developed. The first phase of this framework focuses on developing a time dependent FEM model for the diffusion of the multispecies in concrete while considering the influence of the ionic interaction between the different species. The coupled effect of moisture and multi-ion diffusion is also considered. The second phase is to incorporate the effect of drying shrinkage damage in the diffusion coefficients and coupling parameters of the different species.

\subsubsection{Governing Differential Equations for Multi-Species Diffusion}

This section will address each of the four governing partial differential equations at the core of our solution. The two governing equations for ionic transport processes in non-saturated concrete are based on the principle of mass conservation law. The mass conservation law for each ion in the solution is defined as: 


$$
\frac{\partial C_{i}}{\partial t}=-\nabla J_{i}
$$

in which $C$ is the concentration of the ionic species, $J$ is the ion flux, and subscript $i$ is for species $i$, and $\mathrm{t}$ is the time.

Multi-Species Diffusion

The ionic flux through the saturated medium is defined using the extended Nernst-Planck equation:

$$
J_{i}=-\left(D_{i} \nabla C_{i}+z_{i} D_{i}\left(\frac{F}{R T} \nabla \Phi\right) C_{i}\right)
$$

where $\mathrm{F}$ is Faraday's constant, $\mathrm{R}$ is the universal gas constant, $\mathrm{T}$ is the temperature, $\mathrm{z}$ is the valence number of the species, and $\Phi$ is the applied electrical potential. The two terms in the right-hand side of Eq. 63 represent two different mechanisms. The first mechanism is the movement of ions induced by the concentration gradient (Fick's law), and the second mechanism is the effect of the electrical field. (At this stage, the model does not account for the effect of the chemical activity). To solve the electrical potential, another relation is needed to evaluate the electrical potential induced by the movement of the ions. In this work, the electro-neutrality condition will be used to solve the electrical potential.

$$
\sum_{i=1}^{n} C_{i} z_{i}=0
$$

Substituting Eq.(95) into Eq.(94), the expression for the electro-potential can be expressed as:

$$
\begin{gathered}
J_{i}=\sum_{i=1}^{n} z_{i}\left(-D_{i} \nabla C_{i}-z_{i} D_{i}\left(\frac{F}{R T} \nabla \Phi\right) C_{i}\right)=0 \\
\nabla \Phi=-\frac{\sum_{i=1}^{n} z_{i} D_{i} \nabla C_{i}}{\sum_{i=1}^{n} z_{i}^{2} D_{i} C_{i}} \cdot \frac{R T}{F}
\end{gathered}
$$

\subsubsection{Moisture Diffusion}

The flux of the moisture through the concrete medium is expressed in terms of the pore relative humidity:

$$
J_{H}=\left(-D_{H} \nabla H\right)
$$

Where $\mathrm{H}$ is pore relative humidity $\mathrm{D}_{\mathrm{H}}$ is the humidity diffusivity 


\subsubsection{Coupled Moisture and Multi-Species Diffusion}

To describe the diffusion of the different species in non-saturated concrete. two additional terms were added to the flux equations to consider the coupling effect of the moisture movement on the diffusion of the different species and the effect on the diffusion of the different species on the moisture movement;

$$
\begin{gathered}
J_{i}=\left(-D_{i} \nabla C_{i}-z_{i} D_{i}\left(\frac{F}{R T} \nabla \Phi\right) C_{i}-D_{H-i} \nabla H\right) \\
J_{i}=\left(-D_{H-i} \nabla C_{i}--_{i}-D_{H} \nabla H\right)
\end{gathered}
$$

By substituting Eqs. (99),(100) into Eq. (93), the general governing equations for each individual ionic species can be expressed as:

$$
\begin{gathered}
\frac{\partial w}{\partial t}=\nabla\left(D_{H-i} \nabla C_{i}+D_{H} \nabla H\right) \\
\frac{\partial C_{i t}}{\partial t}=\frac{\partial C_{i t}}{\partial C_{i}} \frac{\partial C_{i}}{\partial t}=\nabla\left(D_{i} \nabla C_{i}+z_{i} D_{i}\left(-\frac{\sum_{i=1}^{n} z_{i} D_{i} \nabla C_{i}}{\sum_{i=1}^{n} z_{i}^{2} D_{i} C_{i}}\right) C_{i}+D_{H-i} \nabla H\right)
\end{gathered}
$$

Considering continuity equations (101),(102) the system of equations to be solved numerically. A finite-element method has been used to compute the concentration profiles of the different species and the electrical field across the sample by applying the Galerkin procedure to the weak forms of governing equations of Equations (101) and (102) the finite element matrix can be obtained as follow:

$$
\left[M^{e}\right]\left\{\dot{C}_{i}\right\}+\left[K^{e}\right]\left\{C_{i}\right\}=0
$$

Where

$$
\begin{aligned}
& {\left[M^{e}\right]=\int\left[N^{T}\right][m][N] d \Omega} \\
& {\left[K^{e}\right]=\int\left[B^{T}\right][D][B] d \Omega}
\end{aligned}
$$

where $[\mathrm{Me}]$, is the capacity matrix; and, [Ke] is the conductivity matrix, [m] and [D] can be expressed as 


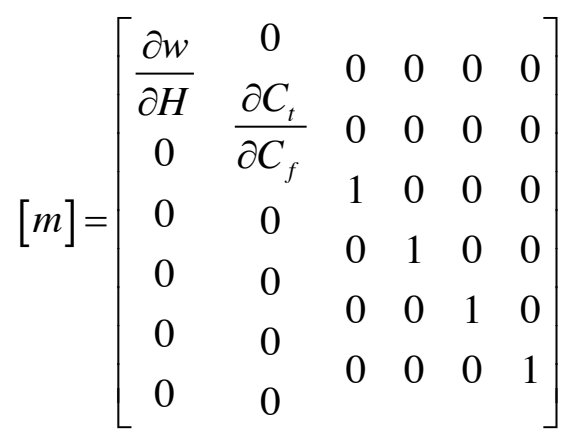

$$
\begin{aligned}
& {[D]=\left[\begin{array}{cccccc}
D_{H-H} & D_{H-C l} & D_{H-N a} & D_{H-K} & D_{H-O H} & D_{H-C a} \\
D_{C l-H} & D_{C l-C l} & D_{C l-N a} & D_{C l-K} & D_{C l-O H} & D_{C l-C a} \\
D_{N a-H} & D_{N a-C l} & D_{N a-N a} & D_{N a-K} & D_{N a-O H} & D_{N a-C a} \\
D_{K-H} & D_{K-C l} & D_{K-N a} & D_{K-K} & D_{K-O H} & D_{K-C a} \\
D_{O H-H} & D_{O H-C l} & D_{O H-N a} & D_{O H-K} & D_{O H-O H} & D_{O H-C a} \\
D_{\mathrm{Ca}-\mathrm{H}} & D_{\mathrm{Ca}-\mathrm{Cl}} & D_{\mathrm{Ca}-\mathrm{Na}} & D_{\mathrm{Ca}-\mathrm{K}} & D_{\mathrm{Ca}-\mathrm{OH}} & D_{\mathrm{Ca}-\mathrm{Ca}}
\end{array}\right]}
\end{aligned}
$$

Considering the Expression and origin of the self-induced electrical field Eq.(97), the fully coupled diffusion coefficients matrix [D] can be expressed as:

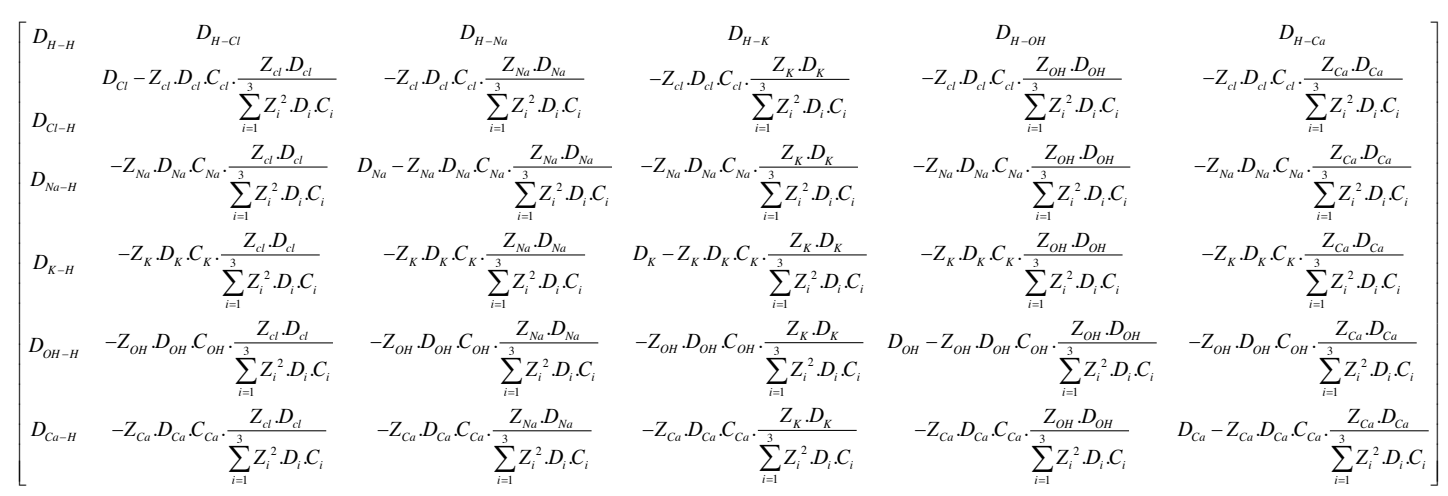

\section{Numerical Example}

The numerical results shown below are for simulations involve six different ionic species ( $\mathrm{RH}$, $\mathrm{Na} \mathrm{Cl}, \mathrm{OH}, \mathrm{K}, \mathrm{Ca}$ ). In this simulations, the geometry of the concrete sample is $3 \mathrm{~cm}$ by $6 \mathrm{~cm}$. The sample is exposed to $3 \% \mathrm{NaCl}$ and $3 \% \mathrm{CaCl}_{2}$ solutions on the top surface; the other boundaries are assumed to be insulated. The concentration of the ions in the concrete pore solution are assumed as follow $\mathrm{RH}=50 \%, \mathrm{~K}=4.90 \mathrm{E}-03 \mathrm{~g} / \mathrm{g}, \mathrm{Na}=1.13 \mathrm{E}-04 \mathrm{~g} / \mathrm{g}$, and $\mathrm{OH}=2.96 \mathrm{E}-$ $03 \mathrm{~g} / \mathrm{g}$. The input data for material parameters are $\mathrm{w} / \mathrm{c}=0.55, \mathrm{gi}=0.65$

The results of the analysis are shown in the Figure 14. The figure show the concentration profiles for all the species at different times of exposure (10 days, 20 days, 50 days, and 100 days) 

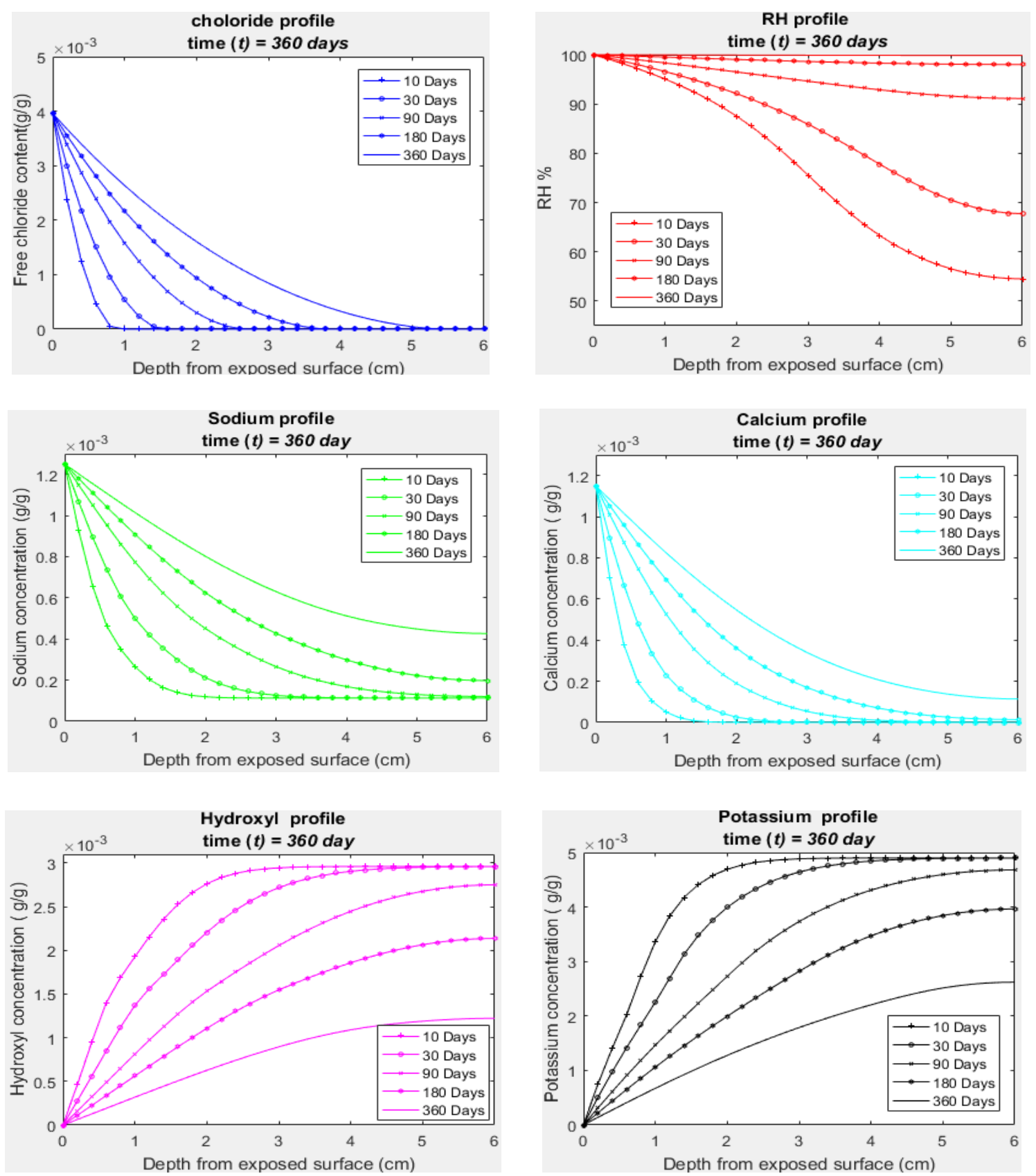

Figure 14: Concentration profiles for all the species at different times of exposure (10 days, 20 days, 50 days, and 100 days) 


\subsection{Implementation of multispecies diffusion coupled with moisture and heat transfer processes in the Grizzly code}

The concrete multispecies diffusion model developed at the University of Colorado, Boulder has been integrated with the transport models for moisture, heat, and species previously developed in INL's Grizzly code (Huang et al. 2015). The Grizzly code previously modeled coupled heat/moisture transfer, Fickian diffusion of dissolved ions and chemical reactions in concrete structures. This capability has been extended to also incorporate species diffusion governed by the extended Nernst-Planck diffusion law.

The coupling of this newly implemented multispecies diffusion model with the existing heat/moisture transfer models and aqueous reaction models in the Grizzly code provides a unique tool for predictive modeling of coupled physical and chemical processes in concrete structures under various environmental conditions.

The underlying physics models are implemented in MOOSE using a set of objects known as Kernels, which define the individual terms in a set of coupled partial differential equations (PDEs). For problems with large numbers of species, a large number of kernels must be defined for the individual terms in the PDE for each species. To provide a simplified user interface for this capability, the Grizzly code provides an Action, which is a MOOSE object that can build up a complex set of models with a simple user interface. This Action is known as

"ReactionNetwork", and automatically sets up all of the necessary objects needed to simulate the free ion species transport in concrete pore water, with options to turn on or off various aqueous speciation reactions and mineral-water precipitation/dissolution reactions.

Figure 15 shows an example of the section of the Grizzly input file used to define this ReactionNetwork. The majority of this input is inactive in this case. The active lines at the top of this figure show the inputs needed to define Nernst-Planck diffusion. The commented out code is included to show how the reactions between species could be included if desired.

The Grizzly code already includes coupled heat/moisture transport models for concrete structures, with a variety of constitutive models for the thermal and moisture transport properties available, as documented in Huang et al. (2015). Grizzly takes advantage of the capabilities provided by the MOOSE framework (Gaston et al. 2009) for solving coupled systems of governing PDEs for the coupled heat transfer, moisture diffusion, mutli-species diffusion processes. These are solved simultaneously using a preconditioned Jacobian Free Newton Krylov (JFNK) method. This provides benefits both for parallel scalability, as the full Jacobian matrix for the system does not need to be formed, and for rapid code development, because off-diagonal Jacobian entries related to coupling between physics do not necessarily need to be defined. 


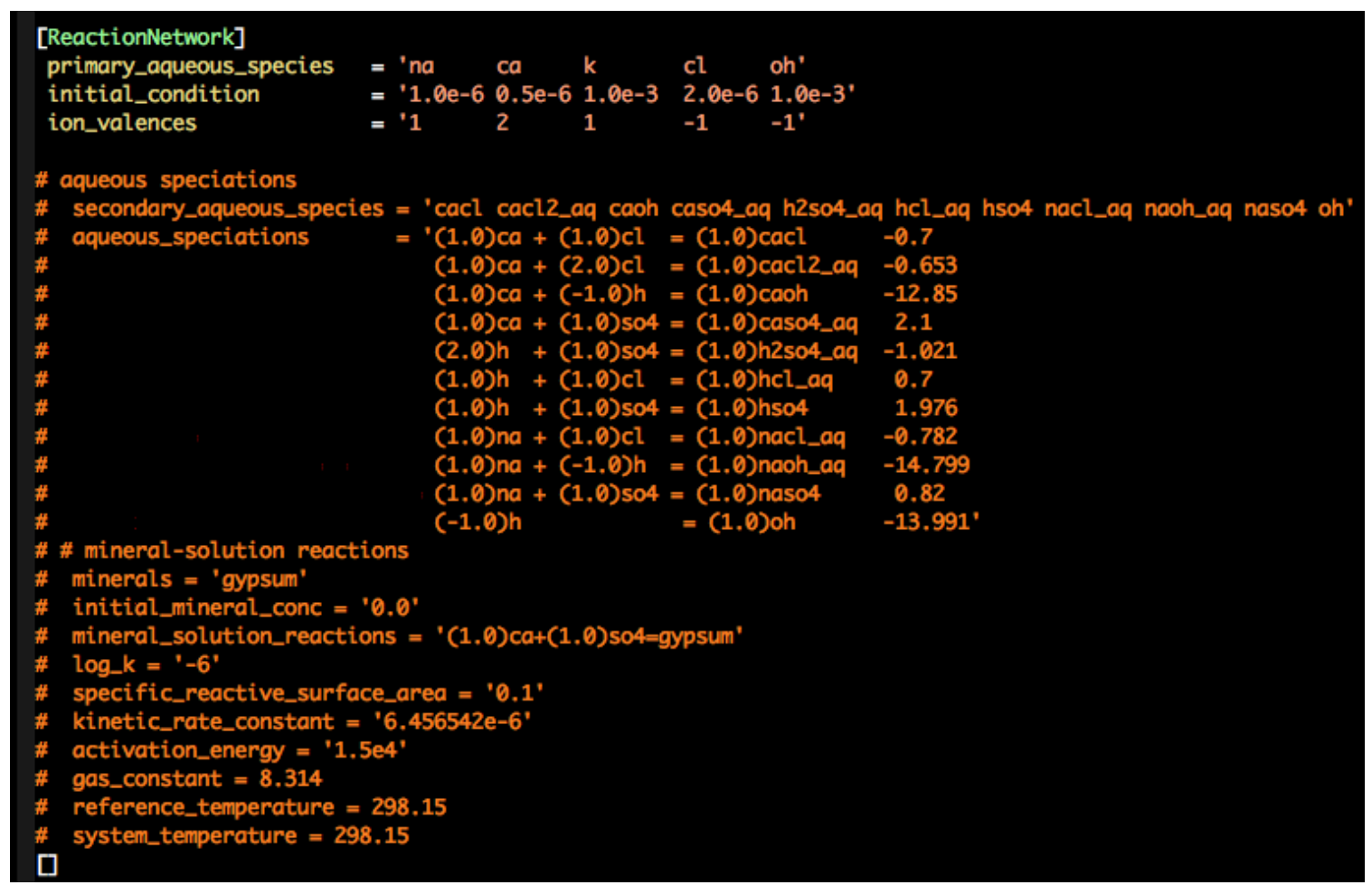

Figure 15: Example input file for setting up multiple ion species in concrete pore water, with aqueous speciation reactions and mineral-water reactions turned off (commented out using the '\#' character).

Figure 16 shows an example simulation where temperature, relative humidity and concentrations of multiple ions in pore water were solved simultaneously. In this example problem, a $3 \mathrm{~cm} \mathrm{x}$ $6 \mathrm{~cm}$ concrete slab with initial relative humidity of 0.1 and temperature $20^{\circ} \mathrm{C}$ is put in contact with a solution of $0.03 \mathrm{M} \mathrm{CaCl}$ and $0.015 \mathrm{M} \mathrm{NaCl}$ at a temperature of $50^{\circ} \mathrm{C}$ on one end. The concentration profiles of various ion species, the profiles of the relative humidity and temperature are shown in Figure 16. These initial results appear to be reasonable, although this model still needs verification and validation. The thermal diffusion is the fastest diffusion process, so thermal equilibrium with the entire model domain was reached first. Moisture and species diffusion are much slower processes, and require significantly more time to reach equilibrium, as evident in the results shown here. 


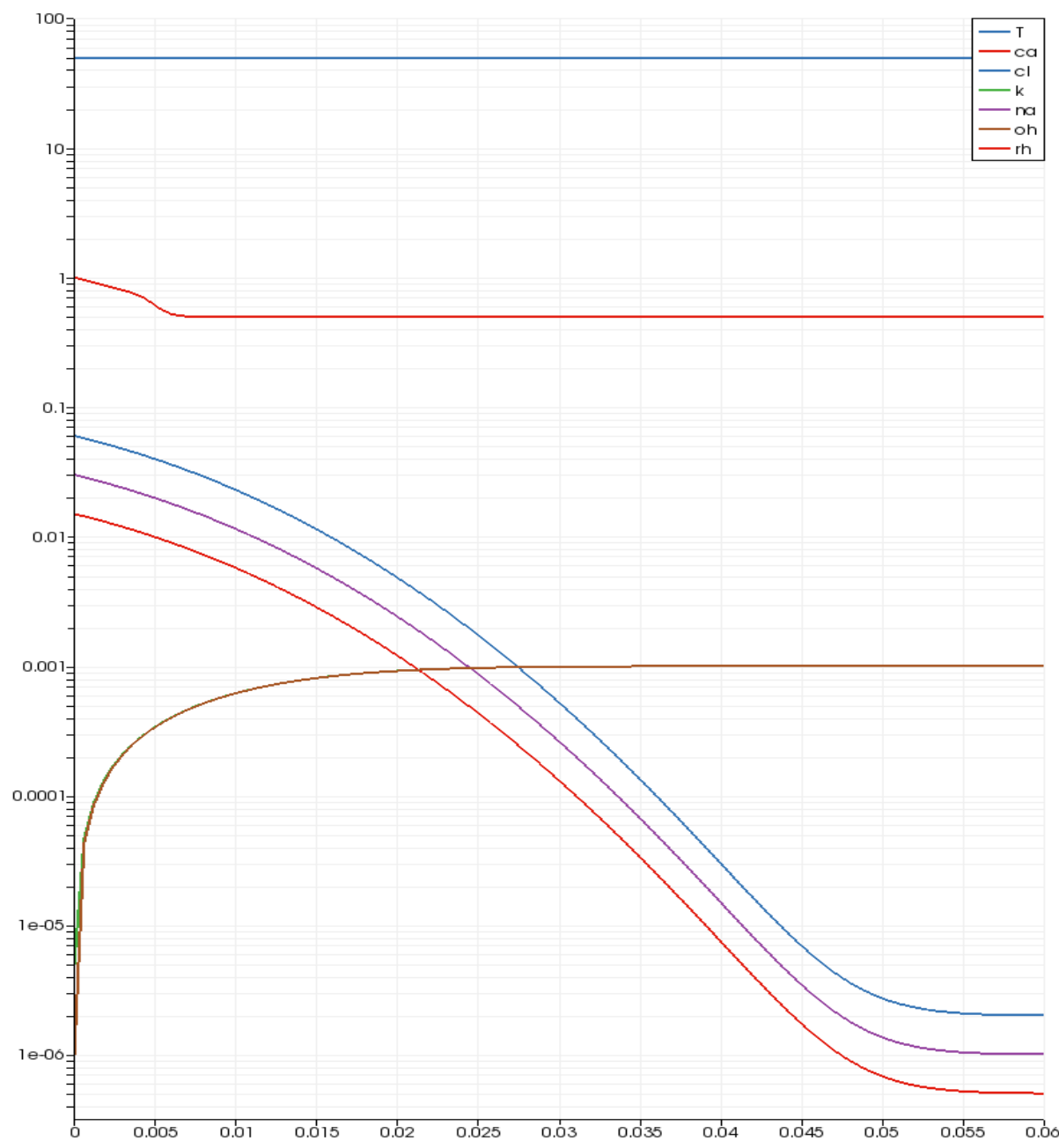

Figure 16: Simulated ion concentration profiles, the temperature profile, and relative humidity profile.

A significant emphasis of future work in this area will be performing validation of these models against results that will be obtained in experiments conducted at the University of Colorado. 


\section{Summary and Future work}

This report has documented the models developed as part of the present project that have been implemented in the BlackBear code:

- A simple damage mechanics model based on the Mazars model

- A damaged plasticity model based on the Lee and Fenves model

- A shrinkage model for concrete

- A general framework for the Nernst-Planck equations for coupled multispecies diffusion

Initial testing of these models has been performed. Experimental work is underway to obtain validation data for the transport models and for the response of large beams subjected to alkalisilica reactions, which will be used to validate the mechanical models developed in this work documented here. As data becomes available from this testing, this will be used to validate the models described here. 


\section{Bibliography}

Ababneh, A. and Xi, Y. (2002). "An experimental study on the effect of the chloride penetration on moisture diffusion in concrete." Materials and Structures 35: 659-664.

Ababneh, A. and Xi, Y. (2003). "An experimental study on the effect of chloride penetration on moisture diffusion in concrete." Materials and Structures: 659-664.

Bazant, Z. P. and Najjar, L. J. (1972). "Nonlinear water diffusion of nonsaturated concrete." Matériaux et Constructions,: 5(25).

Brunauer, S., Skalny, J. and Bodor, E. (1969). "Adsorption on nonporous solids." Journal of Colloid and Interface Science 30(4): 546-552.

Christensen, R. M. (1979). Mechanics of composite materials. New York, Wiley Interscience.

Cicekli, U., Voyiadjis, G. Z. and Al-Rub, R. K. A. (2007). "A plasticity and anisotropic damage model for plain concrete." International Journal of plasticity 23(10): 1874-1900.

Gabet, T., Malécot, Y. and Daudeville, L. (2008). "Triaxial behaviour of concrete under high stresses: Influence of the loading path on compaction and limit states." Cement and Concrete Research 38(3): 403-412.

Gaston, D., Newman, C., Hansen, G. and Lebrun-Grandie, D. (2009). "Moose: A parallel computational framework for coupled systems of nonlinear equations." Nuclear Engineering and Design 239(10): 1768-1778.

Hausmann, D. A. (1967). Steel corrosion in concrete -- how does it occur?, National Association of Corrosion Engineers.

Homan, L., Ababneh, A. N. and Xi, Y. (2016). "The effect of moisture transport on chloride penetration in concrete." Construction and Building Materials 125 1189-1195.

Huang, H., Spencer, B. and Cai, G. (2015). Grizzly model of multi-species reactive diffusion, moisture/heat transfer, and alkalisilica reaction in concrete, Technical Report INL/EXT-15-36425, Idaho National Laboratory.

Imran, I. and Pantazopoulou, S. (2001). "Plasticity model for concrete under triaxial compression." Journal of engineering mechanics 127(3): 281-290.

Lee, J. (1997). "Theory and implementation of plastic-damage model for concrete structures under cyclic and dynamic loading."

Lee, J. and Fenves, G. L. (1998). "Plastic-damage model for cyclic loading of concrete structures." Journal of engineering mechanics 124(8): 892-900.

Lee, J. and Fenves, G. L. (2001). "A return-mapping algorithm for plastic-damage models: 3-d and plane stress formulation." International Journal for Numerical Methods in Engineering 50(2): 487-506.

Lubliner, J., Oliver, J., Oller, S. and Onate, E. (1989). "A plastic-damage model for concrete." International Journal of solids and structures 25(3): 299-326.

Luping, T. and Nilsson, L.-O. (1993). "Chloride binding capacity and binding isotherms of opc pastes and mortars." Cement and concrete research 23(2): 247-253.

Martys, N. S. and Torquato, S. a. B. (1994). "Universal scaling of fluid permeability for sphere packing." Phys. Rev., E,: 50(51), 403-408.

Page, C. L., Short, N. R. and Tarras, E. (1981). "Diffusion of chloride ions in hardened cement paste." Cement and Concrete Research: 11(13), 395-406.

Poinard, C., Malecot, Y. and Daudeville, L. (2010). "Damage of concrete in a very high stress state: Experimental investigation." Materials and Structures 43(1-2): 15-29. 
Rosen, B. W. and Hashin, Z. (1970). "Effective thermal expansion coefficientsand specific heats of composite materials "Imt. J. Eng. Sci 8: 157.

Sfer, D., Carol, I., Gettu, R. and Etse, G. (2002). "Study of the behavior of concrete under triaxial compression." Journal of Engineering Mechanics 128(2): 156-163.

Xi, Y. (1995). "A model for moisture capacities of composite materials - part 1:

Formulation." Computational Materials Science 65-77.

Xi, Y. (1995). "A model for moisture capacities of composite materials part ii: Application to concrete." Computational Materials Science: 78-92.

Xi, Y., Bazant, Z. and Jennings, H. (1994). "Moisture diffusion in cementitious materials: Moisture capacity and diffusion coefficient." Advanced Cement Based Materials: 1, 258-266.

Xi, Y. and Bazant, Z. P. (1999). "Modeling chloride penetration in saturated concrete." Journal of Materials in Civil Engineering: 11 (11), 58-65.

Xi, Y., Bazant, Z. P. and Jennings, H. M. (1994). "Moisture diffusion in cementitious materials : Adsorption isotherm." Advanced Cement Based Materials: 1, 248- 257.

Xi, Y. and Jennings, H. (1997). "Shrinkage of cement paste and concrete modelled by a multiscale effective homogeneous theory." Materials and Structures: 329-339. 